\title{
STABILITY, COHOMOLOGY VANISHING, AND NONAPPROXIMABLE GROUPS
}

\author{
MARCUS DE CHIFFRE ${ }^{1}$, LEV GLEBSKY ${ }^{2}$, ALEXANDER LUBOTZKY ${ }^{3}$ \\ and ANDREAS THOM ${ }^{4}$ \\ ${ }^{1}$ TU Dresden, Germany; \\ email: marcus@dechiffre.dk \\ ${ }^{2}$ L.G., Universidad Autónoma de San Luis Potosí, México; \\ email: glebsky@cactus.iico.uaslp.mx \\ ${ }^{3}$ A.L., Hebrew University, Israel; \\ email: alex.lubotzky@mail.huji.ac.il \\ ${ }^{4}$ TU Dresden, Germany; \\ email: andreas.thom@tu-dresden.de
}

Received 28 March 2019; accepted 20 December 2019

\begin{abstract}
Several well-known open questions (such as: are all groups sofic/hyperlinear?) have a common form: can all groups be approximated by asymptotic homomorphisms into the symmetric groups $\operatorname{Sym}(n)$ (in the sofic case) or the finite-dimensional unitary groups $\mathrm{U}(n)$ (in the hyperlinear case)? In the case of $\mathrm{U}(n)$, the question can be asked with respect to different metrics and norms. This paper answers, for the first time, one of these versions, showing that there exist finitely presented groups which are not approximated by $\mathrm{U}(n)$ with respect to the Frobenius norm $\|T\|_{\text {Frob }}=\sqrt{\sum_{i, j=1}^{n}\left|T_{i j}\right|^{2}}$, $T=\left[T_{i j}\right]_{i, j=1}^{n} \in \mathrm{M}_{n}(\mathbb{C})$. Our strategy is to show that some higher dimensional cohomology vanishing phenomena implies stability, that is, every Frobenius-approximate homomorphism into finite-dimensional unitary groups is close to an actual homomorphism. This is combined with existence results of certain nonresidually finite central extensions of lattices in some simple $p$-adic Lie groups. These groups act on high-rank Bruhat-Tits buildings and satisfy the needed vanishing cohomology phenomenon and are thus stable and not Frobenius-approximated.
\end{abstract}

2010 Mathematics Subject Classification: 22D10 (primary); 39B82 (secondary)

(c) The Author(s) 2020. This is an Open Access article, distributed under the terms of the Creative Commons Attribution licence (http://creativecommons.org/licenses/by/4.0/), which permits unrestricted re-use, distribution, and reproduction in any medium, provided the original work is properly cited. 


\section{Introduction}

Since the very beginning of the study of groups, groups were studied by looking at their orthogonal and unitary representations. It is very natural to relax the notion of a representation and require the group multiplication to be preserved only up to little mistakes in a suitable metric. First variations of this topic appeared already in the work of Turing [52] and later Ulam [53, Ch. VI]. This theme knows many variations, ranging from sofic approximations as introduced by Gromov [26] and operator-norm approximations that appeared in the theory of operator algebras $[10,15]$ to questions related to Connes' Embedding Problem; see [16, 38, 48] for details. In each case, approximation properties of groups are studied relative to a particular class of metric groups. More specifically, let $\Gamma$ be a countable group and let $\left(G_{n}, d_{n}\right)_{n=1}^{\infty}$ be a sequence of metric groups with bi-invariant metrics $d_{n}$. We say that $\Gamma$ is $\left(G_{n}, d_{n}\right)_{n=1}^{\infty}$-approximated if there exists a separating sequence of asymptotic homomorphisms $\varphi_{n}: \Gamma \rightarrow G_{n}$, that is, a sequence of maps $\varphi_{n}$ that becomes multiplicative in the sense that

$$
\lim _{n \rightarrow \infty} d_{n}\left(\varphi_{n}(g h), \varphi_{n}(g) \varphi_{n}(h)\right)=0, \quad \text { for all } g, h \in \Gamma,
$$

which is also separating, that is, $d_{n}\left(\varphi_{n}(g), 1_{G_{n}}\right)$ is bounded away from zero for all $g \neq 1_{\Gamma}$; see Section 1.4 for precise definitions. Several examples of this situation have been studied in the literature (see [3] for a survey):

(i) $G_{n}=\operatorname{Sym}(n)$, the symmetric group on an $n$-point set, with $d_{n}$ the normalized Hamming distance. In this case, $\left(G_{n}, d_{n}\right)_{n=1}^{\infty}$-approximated groups are called sofic; see [26, 38].

(ii) $G_{n}$, an arbitrary finite group equipped with any bi-invariant metric. In this case, approximated groups are called weakly sofic, or $\mathcal{C}$-approximated, depending on a particular restricted family $\mathcal{C}$ of finite groups. An interesting connection to profinite group theory and recent advances can be found in $[25,34]$.

(iii) $G_{n}=\mathrm{U}(n)$, the unitary group on an $n$-dimensional Hilbert space, where the metric $d_{n}$ is induced by the normalized Hilbert-Schmidt norm $\|T\|_{\mathrm{HS}}=$ $\sqrt{n^{-1} \sum_{i, j=1}^{n}\left|T_{i j}\right|^{2}}$. In this case, approximated groups are sometimes called hyperlinear [38].

(iv) $G_{n}=\mathrm{U}(n)$, where the metric $d_{n}$ is induced by the operator norm $\|T\|_{\text {op }}=$ $\sup _{\|v\|=1}\|T v\|$. In this case, groups which are $\left(G_{n}, d_{n}\right)_{n=1}^{\infty}$-approximated groups are called MF; see [15]. 
(v) $G_{n}=\mathrm{U}(n)$, where the metric $d_{n}$ is induced by the unnormalized HilbertSchmidt norm $\|T\|_{\text {Frob }}=\sqrt{\sum_{i, j=1}^{n}\left|T_{i j}\right|^{2}}$, also called Frobenius norm. We will speak about Frobenius-approximated groups in this context.

Note that the approximation properties are local in the sense that only finitely many group elements and their relations have to be considered for fixed $\varphi_{n}$. This is in stark contrast to the uniform situation, which-starting with the work of GroveKarcher-Ruh and Kazhdan [27, 29] —is much better understood; see [14, 17].

Well-known and longstanding problems, albeit in different fields of mathematics, ask if any group exists which is not approximated in either of the above settings. In setting (i), this is Gromov's question whether all groups are sofic $[26,38]$. The similar question in the context of (iii) is closely related to Connes' Embedding Problem [16, 38]. Indeed, the existence of a non-hyperlinear group would answer Connes' Embedding Problem in the negative. In [10], Kirchberg asked whether any stably finite $C^{*}$-algebra is embeddable into a norm ultraproduct of matrix algebras, implying a positive answer to the approximation problem in the sense of (iv) for any group. Recent breakthrough results imply that any amenable group is MF, that is, approximated in the sense of (iv); see [47].

In this paper, we want to introduce a conceptually new technique that allows us to provide groups that are not approximated in the sense of (v) above, that is, we show that there are finitely presented groups which are not approximated by unitary groups $\{\mathrm{U}(n) \mid n \in \mathbb{N}\}$ with their Frobenius norm. Our techniques do not apply directly to the context of (iii), so we cannot say anything conclusive about Connes' Embedding Problem, but since the norms in (iii) and (v) are related by a normalization constant, we believe that we provide a promising new angle of attack.

Before we start out explaining our strategy and some notation, let us state the main results of this article.

THEOREM 1.1. There exist finitely presented groups which are not Frobeniusapproximated.

The groups we construct are central extensions of cocompact lattices in simple $p$-adic Lie groups. Specifically, we can take certain central extensions of $\mathrm{U}(2 n) \cap$ $\operatorname{Sp}(2 n, \mathbb{Z}[i, 1 / p])$ for $n \geqslant 3$ and $p$ a large enough prime.

To prove Theorem 1.1, we use the notion of stability: A group is called $\left(G_{n}\right.$, $d_{n}$ )-stable if every asymptotic homomorphism (not necessarily a separating one) is 'close' to a true homomorphism (see Definition 1.9). Now, if $G_{n}=\mathrm{U}(n)$ and $\Gamma$ is $\left(G_{n}, d_{n}\right)$-approximated and $\left(G_{n}, d_{n}\right)$-stable, one easily deduces that $\Gamma$ must be residually finite. This basic observation suggests a way to find nonapproximated 
groups: find a group $\Gamma$ which is stable but not residually finite. This method has failed so far for two reasons: (1) it is very difficult to prove stability directly and (2) even in the case where stability was proven (see, for example, [4] and the references therein as well as [8]), it was proven in a way that completely classifies asymptotic homomorphism and it is shown that all are close to a genuine homomorphisms. Thus, only groups which are already approximated have been shown to be stable so far. The main technical novelty of our paper is the following theorem which provides a sufficient condition for a group to be Frobeniusapproximated without assuming a priori that the group is approximated.

THEOREM 1.2. Let $\Gamma$ be a finitely presented group such that

$$
H^{2}\left(\Gamma, \mathcal{H}_{\pi}\right)=\{0\}
$$

for every unitary representation $\pi: \Gamma \rightarrow \mathrm{U}\left(\mathcal{H}_{\pi}\right)$. Then, any asymptotic homomorphism $\varphi_{n}: \Gamma \rightarrow \mathrm{U}(n)$ with respect to the Frobenius norm is asymptotically close to a sequence of homomorphisms, that is, $\Gamma$ is Frobeniusstable.

The appearance of vanishing second cohomology groups may look surprising at first sight, but, in fact, one can translate the question of approximating an asymptotic homomorphism by a true homomorphism to a question about splitting an exact sequence. When the norm is submultiplicative (as is the case of the Frobenius norm but not of the normalized Hilbert-Schmidt norm), the kernel of this splitting problem is abelian (see Section 3.4). It is well known that vanishing of the second cohomology with abelian coefficients means splitting of suitable exact sequences and hence is relevant to the question of stability. It is also interesting to observe that the second cohomology has already appeared in the work of Kazhdan [29] in the context of uniform $\varepsilon$-representations (of compact or amenable groups), a concept related to asymptotic representations, albeit essentially different.

Recall that the classical Kazhdan's property (T) is equivalent to the statement that $H^{1}\left(\Gamma, \mathcal{H}_{\pi}\right)=0$ for all unitary representations $\pi: \Gamma \rightarrow \mathrm{U}\left(\mathcal{H}_{\pi}\right)$. We say that a group is $n$-Kazhdan if $H^{n}\left(\Gamma, \mathcal{H}_{\pi}\right)=\{0\}$ for every unitary representation $\pi: \Gamma \rightarrow \mathrm{U}\left(\mathcal{H}_{\pi}\right)$. Theorem 1.2 simply says that every 2-Kazhdan group is Frobenius-stable. Thus, to prove Theorem 1.1, it suffices to find 2-Kazhdan groups which are not residually finite. Now, the seminal work of Garland [24] (as was extended by Ballmann-Światkowski [6] and others-see [31] and Section 4 for details) shows that for every $2 \leqslant r \in \mathbb{N}$ and $p$ large enough, cocompact (arithmetic) lattices in simple $p$-adic Lie groups of rank $r$ are $n$-Kazhdan for every $1 \leqslant n<r$. In fact, a variant of this has been used to give examples of 
groups with property (T), that is, 1-Kazhdan groups, which are not linear (and potentially also not residually finite) by using 'exotic' affine buildings of rank 2 (see [35]). We want to prove the existence of nonresidually finite 2-Kazhdan groups, but there is a catch: as $n=2, r$ should be at least 3, but a well-known result of Tits asserts that for $r \geqslant 3$, there are no 'exotic' buildings of dimension $r$ and the standard ones coming from $p$-adic Lie groups provide lattices which are all residually finite. To work around this point, we imitate a result (and method of proof) of Deligne [18]. Deligne showed that some nonuniform lattices in simple Lie groups (for example, $\operatorname{Sp}(2 n, \mathbb{Z})$ ) have finite central extensions which are not residually finite. Raghunathan [43] extended it also to some cocompact lattices in $\operatorname{Spin}(2, n)$. These examples became famous when Toledo [50] used them to provide examples of fundamental groups of algebraic varieties which are not residually finite. In the last section, we will explain how Deligne's method can be applied also to cocompact lattices in certain $p$-adic Lie groups. Along the way, we use the solution to the congruence subgroup problem for these lattices which was provided by Rapinchuk [45] and Tomanov [51]. This way, we will get finite central extensions of certain cocompact $p$-adic lattices which are themselves not residually finite anymore. Finally, an easy spectral sequence argument shows that a finite (central) extension of an $n$-Kazhdan group is also $n$-Kazhdan. Thus, the nonresidually finite central extensions of the above-mentioned lattices provide the non-Frobenius-approximated group promised in Theorem 1.1.

Along the way in Section 2, we also provide examples of residually finite groups which are not Frobenius-stable and of finitely generated nonresidually finite groups which are Frobenius-approximated. It is currently unclear if maybe all amenable (or even all solvable) groups are Frobenius-approximated. Moreover, it is an open problem to decide if the class of Frobenius-approximated groups is closed under central quotients or under semi-direct products with $\mathbb{Z}$, compare with $[36,49]$.

Added in proof: By extending Theorem 1.2 to more general Banach spaces, the main result of this paper, was extended in [32] from the Frobenius case (that is, $p=2$ ) to all $p$-Schatten norms (for $1<p<\infty$ ).

1.1. Notation. Given any set $S$, we let $\mathbb{F}_{S}$ denote the free group on $S$. For any $R \subseteq \mathbb{F}_{S}$, we let $\langle R\rangle$ denote the normal subgroup generated by $R$ and we let $\langle S \mid R\rangle:=\mathbb{F}_{S} /\langle R\rangle$ be the group with generators $S$ and relations $R$. We use the convention $\mathbb{N}=\{1,2, \ldots\}$. For $n \in \mathbb{N}$, we let $\mathrm{M}_{n}(\mathbb{C})$ denote the complex $n \times n$ matrices and $\mathrm{U}(n) \subseteq \mathrm{M}_{n}(\mathbb{C})$ the group of unitary matrices. The identity matrix is denoted by $1_{n}$.

Recall that an ultrafilter $\mathcal{U}$ on $\mathbb{N}$ is a nontrivial collection of subsets of $\mathbb{N}$ such that (i) $A \in \mathcal{U}, A \subset B$ implies $B \in \mathcal{U}$, (ii) $A, B \in \mathcal{U}$ implies $A \cap B \in \mathcal{U}$, and (iii) 
$A \notin \mathcal{U}$ if and only if $\mathbb{N} \backslash A \in \mathcal{U}$ holds. We say that $\mathcal{U}$ is nonprincipal if $\{n\} \notin \mathcal{U}$ for all $n \in \mathbb{N}$. The existence of nonprincipal ultrafilters on $\mathbb{N}$ is ensured by the Axiom of Choice. We can view a nonprincipal ultrafilter as a finitely additive probability measure defined on all subsets of $\mathbb{N}$, taking only the values $\{0,1\}$ and giving the value 0 to all finite subsets of $\mathbb{N}$.

Throughout the whole paper, we fix a nonprincipal ultrafilter $\mathcal{U}$ on $\mathbb{N}$. Given some statement $P(n)$ for $n \in \mathbb{N}$, we use the wording $P(n)$ holds for most $n \in \mathbb{N}$ as $\{n \in \mathbb{N} \mid P(n)\} \in \mathcal{U}$. Given a bounded sequence $\left(x_{n}\right)_{n \in \mathbb{N}}$ of real numbers, we denote the limit along the ultrafilter by $\lim _{n \rightarrow \mathcal{U}} x_{n} \in(-\infty, \infty)$. Formally, the limit is the unique real number $x$ such that for all $\varepsilon>0$, we have $\{n \in \mathbb{N}$ | $\left.\left|x_{n}-x\right|<\varepsilon\right\} \in \mathcal{U}$. For unbounded sequences, the limit takes a well-defined value in the extended real line $[-\infty, \infty]$.

We adopt the Landau notation; given two sequences $\left(x_{n}\right)_{n \in \mathbb{N}}$ and $\left(y_{n}\right)_{n \in \mathbb{N}}$ of non-negative real numbers, we write $x_{n}=O_{\mathcal{U}}\left(y_{n}\right)$ if there exists $C>0$ such that $x_{n} \leqslant C y_{n}$ for most $n \in \mathbb{N}$ and $x_{n}=o_{\mathcal{U}}\left(y_{n}\right)$ if there exists a third sequence $\left(\varepsilon_{n}\right)_{n \in \mathbb{N}}$ of non-negative real numbers such that $\lim _{n \rightarrow \mathcal{U}} \varepsilon_{n}=0$ and $x_{n}=\varepsilon_{n} y_{n}$.

1.2. Unitarily invariant norms. Recall that a norm $\|\cdot\|$ on $\mathrm{M}_{d}(\mathbb{C})$ is called unitarily invariant if

$$
\|U A V\|=\|A\|,
$$

for all $A \in \mathrm{M}_{d}(\mathbb{C})$ and $U, V \in \mathrm{U}(d)$.

Some important examples of unitarily invariant norms are the operator norm $\|T\|_{\text {op }}=\sup _{\|v\|=1}\|T v\|$, the Frobenius norm $\|T\|_{\text {Frob }}=\operatorname{Tr}\left(T^{*} T\right)^{1 / 2}=$ $\sqrt{\sum_{i, j}^{n}\left|T_{i j}\right|^{2}}$ (also known as the unnormalized Hilbert-Schmidt norm), and the normalized Hilbert-Schmidt norm (or 2-norm) given by $\|T\|_{\mathrm{HS}}=\frac{1}{\sqrt{n}}\|T\|_{\text {Frob }}$ for $T \in \mathrm{M}_{n}(\mathbb{C})$. Here, $T^{*}$ denotes the adjoint matrix and $T$ is called self-adjoint if $T=T^{*}$. The matrix $T$ is called unitary if $T T^{*}=T^{*} T=1_{n}$. We recall some basic and well-known facts about unitarily invariant norms (see, for example, [2] for a thorough introduction to the subject). For $T \in \mathrm{M}_{n}(\mathbb{C})$, we set $|T|=\left(T^{*} T\right)^{1 / 2}$. For self-adjoint matrices $A, B$, we write $A \leqslant B$ if $B-A$ is positive semidefinite, that is, if $B-A$ has only non-negative eigenvalues.

Proposition 1.3. Let $A, B, C \in \mathrm{M}_{d}(\mathbb{C})$. Then, for any unitarily invariant norm, it holds that

(1) $\|A B C\| \leqslant\|A\|_{\text {op }}\|B\|\|C\|_{\text {op }}$,

(2) $\|A\|=\left\|A^{*}\right\|=\||A|\|$,

(3) if $A$ and $B$ are positive semidefinite matrices and $A \leqslant B$, then $\|A\| \leqslant\|B\|$. 
Proposition 1.4. Let $A \in \mathrm{U}(d)$. Then there is a unitary $B \in \mathrm{U}(d)$ such that $B^{2}=1$ and

$$
\|B-A\| \leqslant\left\|1_{d}-A^{2}\right\|,
$$

for all unitarily invariant norms.

Proof. By unitary invariance, we may assume that $A$ is a diagonal matrix, which we denote $A=\operatorname{diag}\left(a_{1}, \ldots, a_{d}\right)$. Let

$$
b_{j}:= \begin{cases}1 & \text { if } \operatorname{Re}\left(a_{j}\right) \geqslant 0, \\ -1 & \text { if } \operatorname{Re}\left(a_{j}\right)<0 .\end{cases}
$$

One readily sees that $\left|b_{j}-a_{j}\right| \leqslant\left|1-a_{j}\right|\left|-1-a_{j}\right|=\left|1-a_{j}^{2}\right|$. Thus, $B=\operatorname{diag}\left(b_{1}\right.$, $\left.\ldots, b_{d}\right)$ is a self-adjoint unitary, and by Proposition 1.3 (3),

$$
\|B-A\|=\||B-A|\| \leqslant\left\|\left|1_{d}-A^{2}\right|\right\|=\left\|1_{d}-A^{2}\right\| .
$$

A second property that is important to us is submultiplicativity, that is, $\|A B\| \leqslant$ $\|A\|\|B\|$ for all $A, B \in \mathrm{M}_{d}(\mathbb{C})$. This property turns $\left(\mathrm{M}_{d}(\mathbb{C}),\|\cdot\|\right)$ into a Banach algebra. The operator norm and the Frobenius norm enjoy this property, but the normalized Hilbert-Schmidt norm does not.

1.3. Ultraproducts. We will need the definition of the ultraproduct of Banach spaces and metric groups, respectively. First, let $\left(V_{n}\right)_{n \in \mathbb{N}}$ be a sequence of Banach spaces. Consider the $\ell^{\infty}$-direct product $\prod_{n \in \mathbb{N}} V_{n}$ (that is, the Banach space of bounded sequences $\left(v_{n}\right)_{n \in \mathbb{N}}$ with $\left.v_{n} \in V_{n}\right)$ and the closed subspace of null sequences

$$
I:=\left\{\left(v_{n}\right)_{n \in \mathbb{N}} \in \prod_{n \in \mathbb{N}} V_{n} \mid \lim _{n \rightarrow \mathcal{U}}\left\|v_{n}\right\|_{V_{n}}=0\right\} .
$$

We define the ultraproduct Banach space by

$$
\prod_{n \rightarrow \mathcal{U}}\left(V_{n},\|\cdot\|_{V_{n}}\right):=\prod_{n \in \mathbb{N}} V_{n} / I \text {. }
$$

As the name suggests, the ultraproduct Banach space is itself a Banach space with the norm induced by $\left\|\left(x_{n}\right)_{n \in \mathbb{N}}\right\|=\lim _{n \rightarrow \mathcal{U}}\left\|x_{n}\right\|_{V_{n}}$ for $\left(x_{n}\right)_{n \in \mathbb{N}} \in \prod_{n \in \mathbb{N}} V_{n}$. Moreover, if the $V_{n}$ are all Banach algebras, $C^{*}$-algebras or Hilbert spaces, so is the ultraproduct.

Let $\left(G_{n}\right)_{n \in \mathbb{N}}$ be a family of groups, all equipped with bi-invariant metrics $d_{n}$. In this case, the subgroup

$$
N=\left\{\left(g_{n}\right)_{n \in \mathbb{N}} \in \prod_{n \in \mathbb{N}} G_{n} \mid \lim _{n \rightarrow \mathcal{U}} d_{n}\left(g_{n}, 1_{G_{n}}\right)=0\right\}
$$


of the direct product $\prod_{n \in \mathbb{N}} G_{n}$ is normal, so we can define the metric ultraproduct

$$
\prod_{n \rightarrow \mathcal{U}}\left(G_{n}, d_{n}\right):=\prod_{n \in \mathbb{N}} G_{n} / N .
$$

Note that, in contrast to the Banach space definition, we do not require the sequences to be bounded. It is worth noting (albeit not relevant for our purposes) that the bi-invariant metric

$$
d\left(\left(g_{n}\right)_{n \in \mathbb{N}},\left(h_{n}\right)_{n \in \mathbb{N}}\right)=\lim _{n \rightarrow \mathcal{U}} \min \left\{d_{n}\left(g_{n}, h_{n}\right), 1\right\}, \quad g_{n}, h_{n} \in G_{n}
$$

on $\prod_{n \in \mathbb{N}} G_{n}$ induces a bi-invariant metric on $\prod_{n \rightarrow \mathcal{U}}\left(G_{n}, d_{n}\right)$.

The above definitions will be relevant to us in the following setting. Let $\left(k_{n}\right)_{n \in \mathbb{N}}$ be a sequence of natural numbers and consider the family of matrix algebras $V_{n}:=$ $\mathrm{M}_{k_{n}}(\mathbb{C})$ equipped with some unitarily invariant, submultiplicative norms $\|\cdot\|_{n}$. We usually omit the index and denote all the norms by $\|\cdot\|$. Let $G_{n}:=\mathrm{U}\left(k_{n}\right)$, equipped with the metrics $\operatorname{dist}_{\|\cdot\|_{n}}(g, h)=\|g-h\|_{n}, g, h \in G_{n}$ induced from the norms. We consider the ultraproduct Banach space

$$
\mathrm{M}_{\mathcal{U}}^{\|\cdot\|}:=\prod_{n \rightarrow \mathcal{U}}\left(\mathrm{M}_{k_{n}}(\mathbb{C}),\|\cdot\|_{n}\right)
$$

and the metric ultraproduct

$$
\mathrm{U}_{\mathcal{U}}^{\|\cdot\|}:=\prod_{n \rightarrow \mathcal{U}}\left(\mathrm{U}\left(k_{n}\right), \operatorname{dist}_{\|\cdot\|_{n}}\right)
$$

By submultiplicativity of the norms, we see that if $u_{n} \in \mathrm{U}\left(k_{n}\right)$ satisfying $\lim _{n \rightarrow \mathcal{U}} \operatorname{dist}_{\|\cdot\|_{n}}\left(u_{n}, 1_{k_{n}}\right)=0$, then

$$
\lim _{n \rightarrow \mathcal{U}}\left\|u_{n} T_{n}-T_{n}\right\|_{n} \leqslant \lim _{n \rightarrow \mathcal{U}}\left\|u_{n}-1_{k_{n}}\right\|_{n} \cdot\left\|T_{n}\right\|_{n}=0,
$$

for all bounded sequences $T_{n} \in \mathrm{M}_{k_{n}}(\mathbb{C})$. Thus, left multiplication by $u_{n}$ induces a continuous left action of $\mathrm{U}_{\mathcal{U}}^{\|\cdot\|}$ on $\mathbf{M}_{\mathcal{U}}^{\|\cdot\|}$. By unitary invariance of the norms, we see that this action is isometric. Similarly, we have a right action by right multiplication and another left action by conjugation-both of them isometric.

1.4. Asymptotic homomorphisms. In this section, we let $\Gamma=\langle S \mid R\rangle$ be a fixed finitely presented group (that is, $S$ and $R$ are finite) and we let $\mathcal{C}$ be a class of groups, all equipped with bi-invariant metrics. Any map $\varphi: S \rightarrow G$, for some $G \in \mathcal{C}$, uniquely determines a homomorphism $\mathbb{F}_{S} \rightarrow G$ which we will also denote by $\varphi$. 
Definition 1.5. Let $G \in \mathcal{C}$ and let $\varphi, \psi: S \rightarrow G$ be maps. The defect of $\varphi$ is defined by

$$
\operatorname{def}(\varphi):=\max _{r \in R} d_{G}\left(\varphi(r), 1_{G}\right) .
$$

The distance between $\varphi$ and $\psi$ is defined by

$$
\operatorname{dist}(\varphi, \psi)=\max _{s \in S} d_{G}(\varphi(s), \psi(s)) .
$$

The homomorphism distance of $\varphi$ is defined by

$$
\operatorname{HomDist}(\varphi):=\inf _{\pi \in \operatorname{Hom}(\Gamma, G)} \operatorname{dist}\left(\varphi,\left.\pi\right|_{S}\right) .
$$

Definition 1.6. A sequence of maps $\varphi_{n}: S \rightarrow G_{n}$, for $G_{n} \in \mathcal{C}$, is called an asymptotic homomorphism if $\lim _{n \rightarrow \mathcal{U}} \operatorname{def}\left(\varphi_{n}\right)=0$.

We will mainly be concerned with finite-dimensional asymptotic representations, that is, asymptotic homomorphisms with respect to the class of unitary groups $\mathrm{U}(n)$ on finite-dimensional Hilbert spaces, equipped with the metrics

$$
d(T, S)=\|T-S\|, \quad T, S \in \mathrm{U}(n),
$$

coming from some family of unitarily invariant norms $\|\cdot\|$. The class of finitedimensional unitary groups with metrics coming from $\|\cdot\|_{\mathrm{op}},\|\cdot\|_{\text {Frob }}$ and $\|\cdot\|_{\mathrm{HS}}$ are denoted as $\mathrm{U}_{\mathrm{op}}, \mathrm{U}_{\text {Frob }}$, and $\mathrm{U}_{\mathrm{HS}}$.

We might also find the need to quantify the above definition.

DeFINITION 1.7. Let $\varepsilon>0$ and $G \in \mathcal{C}$. An $\varepsilon$-almost homomorphism is a map $\varphi: S \rightarrow G$ such that $\operatorname{def}(\varphi) \leqslant \varepsilon$.

In the literature, there are many different (inequivalent) notions of 'almost', 'asymptotic', and 'quasi-' homomorphisms. If one would be precise, the above notion of asymptotic homomorphism could be called a local, discrete asymptotic homomorphism. Local, since we are only interested in the behavior of $\varphi_{n}$ on the set of relations $R$ (compare with the uniform situation [14]), and discrete, because the family of homomorphisms is indexed by the natural numbers.

Definition 1.8. Let $G_{n} \in \mathcal{C}, n \in \mathbb{N}$. Two sequences $\varphi_{n}, \psi_{n}: S \rightarrow G_{n}$ are called (asymptotically) equivalent if $\lim _{n \rightarrow \mathcal{U}} \operatorname{dist}\left(\varphi_{n}, \psi_{n}\right)=0$.

If an asymptotic homomorphism $\left(\varphi_{n}\right)_{n \in \mathbb{N}}$ is equivalent to a sequence of genuine representations, we call $\left(\varphi_{n}\right)_{n \in \mathbb{N}}$ trivial. 
We will now come to two central notions that we study in this paper, the notions of stability and approximability by a class of metric groups.

Definition 1.9. The group $\Gamma$ is called $\mathcal{C}$-stable if all asymptotic homomorphisms are equivalent to a sequence of homomorphisms, that is,

$$
\lim _{n \rightarrow \mathcal{U}} \operatorname{HomDist}\left(\varphi_{n}\right)=0,
$$

for all $\varphi_{n}: S \rightarrow G_{n}, G_{n} \in \mathcal{C}, n \in \mathbb{N}$ with $\lim _{n \rightarrow \mathcal{U}} \operatorname{def}\left(\varphi_{n}\right)=0$.

Definition 1.10. A finitely presented group $\Gamma=\langle S \mid R\rangle$ is called $\mathcal{C}$ approximated, if there exists an asymptotic homomorphism $\varphi_{n}: S \rightarrow G_{n}$, $G_{n} \in \mathcal{C}, n \in \mathbb{N}$ such that

$$
\lim _{n \rightarrow \mathcal{U}} d_{n}\left(\varphi_{n}(x), 1_{G_{n}}\right)>0, \quad \text { for all } x \in \mathbb{F}_{S} \backslash\langle R\rangle .
$$

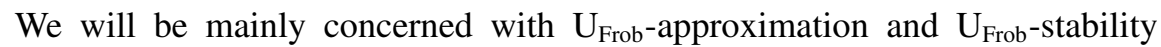
in this paper and, for convenience, we will often just speak about Frobenius approximation and Frobenius stability in this context.

Definition 1.11. A group $\Gamma$ is called residually $\mathcal{C}$ if, for all $x \in \Gamma \backslash\left\{1_{\Gamma}\right\}$, there is a homomorphism $\pi: \Gamma \rightarrow G$ for some $G \in \mathcal{C}$ such that $\pi(x) \neq 1_{G}$.

The following proposition (see [25] or [4]) is evident from the definitions, nevertheless a central observation in our work.

Proposition 1.12. Let $\Gamma$ be a finitely presented group. If $\Gamma$ is $\mathcal{C}$-stable and $\mathcal{C}$-approximated group, then it must be residually $\mathcal{C}$. In particular, if the class $\mathcal{C}$ consists of finite-dimensional unitary groups, any finitely presented, $\mathcal{C}$-stable, and $\mathcal{C}$-approximated group is residually finite.

We finish this section with a basic lemma. The important part in the statement of the lemma is that $K_{r}$ does not depend on $\varphi$.

LEMMA 1.13. For all $r \in\langle R\rangle$, there is a constant $K_{r}$ such that for all groups $G$ with a bi-invariant metric and all maps $\varphi: S \rightarrow G$, it holds that

$$
d_{G}\left(\varphi(r), 1_{G}\right) \leqslant K_{r} \operatorname{def}(\varphi) .
$$

Proof. If $r \in\langle R\rangle$, we can determine $r_{1}, \ldots, r_{k} \in R \cup R^{-1}$ and $x_{1}, \ldots, x_{k} \in \mathbb{F}_{S}$ such that

$$
r=x_{1} r_{1} x_{1}^{-1} x_{2} r_{2} x_{2}^{-1} \cdots x_{k} r_{k} x_{k}^{-1}
$$


Note that by bi-invariance

$$
d_{G}\left(\varphi\left(r_{j}\right), 1_{G}\right)=d_{G}\left(\varphi\left(r_{j}^{-1}\right), 1_{G}\right) \leqslant \operatorname{def}(\varphi)
$$

for all $j$. Thus, using bi-invariance again, we get

$$
\begin{aligned}
d_{G}\left(\varphi(r), 1_{k_{n}}\right) & =d_{G}\left(\varphi\left(x_{1} r_{1} x_{1}^{-1}\right) \cdots \varphi\left(x_{k} r_{k} x_{k}^{-1}\right), 1_{G}\right) \\
& \leqslant \sum_{j=1}^{k} d_{G}\left(\varphi\left(x_{j}\right) \varphi\left(r_{j}\right) \varphi\left(x_{j}\right)^{-1}, 1_{G}\right) \\
& =\sum_{j=1}^{k} d_{G}\left(\varphi\left(r_{j}\right), 1_{G}\right) \\
& \leqslant k \cdot \operatorname{def}(\varphi) .
\end{aligned}
$$

So letting $K_{r}=k$, we are done.

1.5. Group cohomology. We recall one construction of group cohomology. We primarily need the second cohomology of a group with coefficients in a unitary representation, but for completeness, we give a more general definition. Let $\Gamma$ be any group and let $V$ be a $\Gamma$-module, that is, an abelian group together with a (left) action $\pi$ of $\Gamma$ on $V$. We consider the chain complex $C^{n}(\Gamma, V), n \geqslant 1$, which is the set of functions from $\Gamma^{n}$ to $V$ together with the coboundary operator,

$$
d=d^{n}: C^{n}(\Gamma, V) \rightarrow C^{n+1}(\Gamma, V),
$$

defined by

$$
\begin{aligned}
d^{n}(f)\left(g_{1}, \ldots, g_{n+1}\right)= & \pi\left(g_{1}\right) f\left(g_{2}, \ldots, g_{n+1}\right) \\
& +\sum_{j=1}^{n}(-1)^{j} f\left(g_{1}, \ldots, g_{j} g_{j+1}, \ldots, g_{n+1}\right) \\
& +(-1)^{n+1} f\left(g_{1}, \ldots, g_{n}\right) .
\end{aligned}
$$

We also let $C^{0}(\Gamma, V)=V$ and $d^{0}(v)(g)=\pi(g) v-v$ for $v \in V, g \in \Gamma$. Thus, for $n \geqslant 0$, we define the $n$-coboundaries to be $B^{n}(\Gamma, V)=\operatorname{Im}\left(d^{n-1}\right)$ (with $B^{0}(\Gamma$, $V)=\{0\})$ and the $n$-cocycles to be $Z^{n}(\Gamma, V)=\operatorname{ker}\left(d^{n}\right)$. One checks that $B^{n}(\Gamma$, $V) \subseteq Z^{n}(\Gamma, V)$, so we can define the $n$th cohomology to be

$$
H^{n}(\Gamma, V)=Z^{n}(\Gamma, V) / B^{n}(\Gamma, V) .
$$

Recall that, given an extension of groups

$$
1 \rightarrow V \stackrel{i}{\rightarrow} \hat{\Gamma} \stackrel{q}{\rightarrow} \Gamma \rightarrow 1
$$


where $V$ is abelian, there is an action of $\Gamma$ on $V$ induced by the conjugation action of $\hat{\Gamma}$ on $i(V)$. Fixing any section $\sigma: \Gamma \rightarrow \hat{\Gamma}$ (with $\sigma\left(1_{\Gamma}\right)=1_{\hat{\Gamma}}$ ) of the quotient $q$, we can define a map $f: \Gamma \times \Gamma \rightarrow V$ as the solution to

$$
i(f(g, h))=\sigma(g) \sigma(h) \sigma(g h)^{-1},
$$

for $g, h \in \Gamma$. It is straightforward to check that $f \in Z^{2}(\Gamma, V, \pi)$ and $f \in$ $B^{2}(\Gamma, V, \pi)$ exactly when the extension splits, that is, there is a homomorphism $p: \Gamma \rightarrow \hat{\Gamma}$ such that $q \circ p=\mathrm{id}_{\Gamma}$.

Assume now that $\Gamma$ is countable and $V$ is a Banach space with norm $\|\cdot\|$, then we can define a separating family of seminorms on $C^{n}(\Gamma, V)$ by

$$
\|f\|_{F}=\max _{g \in F}\|f(g)\|,
$$

for $f \in C^{n}(\Gamma, V)$ and finite $F \subseteq \Gamma^{n}$. It is easy to see that with respect to this family, $C^{n}(\Gamma, V)$ is a Fréchet space (one can even take $\|\cdot\|_{\{x\}}, x \in \Gamma^{n}$ as separating family) and if $\Gamma$ acts on $V$ by isometries, the map $d^{n}$ is bounded.

\section{Some examples of non-Frobenius-stable groups}

Part of our aim is to provide a large class of Frobenius-stable groups, but let us start out by giving examples of well-known groups that are not stable. Specifically, we show that $\mathbb{Z}^{2}$ and the Baumslag-Solitar group BS(2,3) are not Frobenius-stable by giving concrete examples of asymptotic representations that are not equivalent to genuine representations. We also exploit the latter example to provide an example of an Frobenius-approximated, nonresidually finite group; see Section 2.3.

2.1. $\mathbb{Z}^{2}$ is not Frobenius-stable. In [54], Voiculescu proved that the matrices

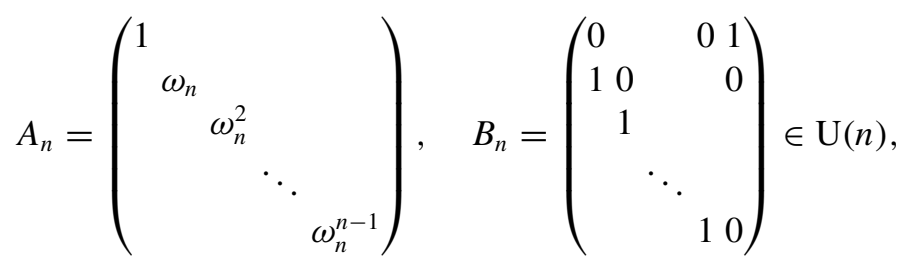

where $\omega_{n}:=\exp \left(\frac{2 \pi i}{n}\right), n \in \mathbb{N}$, define a nontrivial $\|\cdot\|_{\text {op }}$-asymptotic representation of $\mathbb{Z}^{2}=\left\langle a, b, \mid a b a^{-1} b^{-1}\right\rangle$ by $\varphi_{n}(a)=A_{n}$ and $\varphi_{n}(b)=B_{n}$. More precisely,

$$
\operatorname{def}_{\|\cdot\|_{\mathrm{op}}}\left(\varphi_{n}\right)=\left\|A_{n} B_{n} A_{n}^{*} B_{n}^{*}-1_{n}\right\|_{\text {op }}=\left|\omega_{n}-1\right|=O_{\mathcal{U}}\left(\frac{1}{n}\right)
$$


but

$$
\text { HomDist }_{\|\cdot\|_{\text {op }}}\left(\varphi_{n}\right) \geqslant \sqrt{2-\left|1-\omega_{n}\right|}-1
$$

(see also [22, 54]). By the inequalities $\|T\|_{\text {op }} \leqslant\|T\|_{\text {Frob }} \leqslant n^{1 / 2}\|T\|_{\text {op }}$ for $T \in \mathrm{U}(n)$, we conclude that

$$
\operatorname{def}_{\|\cdot\|_{\text {Frob }}}\left(\varphi_{n}\right)=O_{\mathcal{U}}\left(n^{-1 / 2}\right)
$$

and

$$
\text { HomDist }_{\|\cdot\|_{\text {Frob }}}\left(\varphi_{n}\right) \geqslant \sqrt{2-\left|1-\omega_{n}\right|}-1,
$$

so $\varphi_{n}$ is also a nontrivial $\|\cdot\|_{\mathrm{Frob}}$-asymptotic representation. In particular, $\mathbb{Z}^{2}$ is neither $\mathrm{U}_{\mathrm{op}}$ - nor Frobenius-stable. It is worth noting that $\mathbb{Z}^{2}$ actually is $\mathrm{U}_{\mathrm{HS}}$-stable, which, for example, follows from unpublished observations from the second author: Almost commuting matrices with respect to normalized Hilbert-Schmidt norm.

2.2. $\mathbf{B S}(2,3)$ is not Frobenius-stable. We now turn our attention to the Baumslag-Solitar group $\mathrm{BS}(2,3)=\left\langle a, b \mid b^{-1} a^{2} b a^{-3}\right\rangle$; see [7] for the original reference. By definition, the generators satisfy the equation

$$
b^{-1} a^{2} b=a^{3} .
$$

It is also well known and not hard to check that the generators do not satisfy

$$
a b^{-1} a b=b^{-1} a b a .
$$

Indeed, this follows easily from the description of $\mathrm{BS}(2,3)$ as an $\mathrm{HNN}$-extension of $\mathbb{Z}$. On the other hand, we recall the following.

Proposition 2.1 (Baumslag-Solitar [7]). Let $\Gamma$ be a residually finite group. If $a, b \in \Gamma$ satisfy (2.1), then they also satisfy (2.2).

Proof. Indeed, if $a$ has finite order and $a^{2}$ is conjugate to $a^{3}$, then the order of $a$ cannot be even. Thus, $b^{-1} a b$ is a power of $b^{-1} a^{2} b=a^{3}$. We conclude that $a$ and $b^{-1} a b$ commute.

By Mal'cev's theorem, we immediately obtain the following consequence.

COROLlaRY 2.2. Let $a, b$ be unitary matrices. If $a, b \in \Gamma$ satisfy (2.1), then they also satisfy (2.2).

This last corollary can also be proven directly by linear algebra methods; see [23] where some quantitative aspects of operator-norm approximability of 
$\mathrm{BS}(2,3)$ were studied. By Corollary 2.2 , in order to show that $\mathrm{BS}(2,3)$ is nonstable, it suffices to find a sequence of pairs of unitary matrices that $\|\cdot\|_{\mathrm{Frob}^{-}}$ asymptotically satisfy Equation (2.1) but are far from satisfying Equation (2.2). The study of approximation properties of $\operatorname{BS}(2,3)$ goes back to Rădulescu [42], where the focus was more on approximation in the (normalized) Hilbert-Schmidt norm. We are now going to prove the following result.

THEOREM 2.3. The group $\mathrm{BS}(2,3)$ is not Frobenius-stable.

The theorem is a direct consequence of the following lemma.

LEMMA 2.4. There exist $A_{n}, B_{n} \in \mathrm{U}(6 n)$ such that

- $\left\|B_{n}^{-1} A_{n}^{2} B_{n}-A_{n}^{3}\right\|_{\text {Frob }}=O_{\mathcal{U}}\left(\frac{1}{n}\right)$

- $\left\|A_{n} B_{n}^{-1} A_{n} B_{n}-B_{n}^{-1} A_{n} B_{n} A_{n}\right\|_{\text {Frob }}=\sqrt{6 n}-O_{\mathcal{U}}(1)$.

Proof. We will omit the index and write $A=A_{n}$ and $B=B_{n}$. Let $\omega=\exp \left(\frac{2 \pi}{6 n}\right)$ and consider a $6 n$-dimensional Hilbert space $\mathcal{H}$ with orthonormal basis $v[0], v[1]$, $\ldots, v[6 n-1]$. Define $A \in \mathrm{U}(6 n)$ as $A v[j]=\omega^{j} v[j]$ (that is, $A$ is $A_{6 n}$ from the previous example). We plan to decompose $\mathcal{H}$ as a direct sum in two ways $\mathcal{H}=\bigoplus_{j=0}^{n-1} \mathcal{S}[j]$ and $\mathcal{H}=\bigoplus_{j=0}^{n-1} \mathcal{C}[j]$ such that each $\mathcal{S}[j]$ and each $\mathcal{C}[j]$ is sixdimensional and the restriction of $A^{2}$ to $\mathcal{S}[j]$ as well as restriction of $A^{3}$ to $\mathcal{C}[j]$ act approximately as multiplication by $\omega^{6 j}$. (The letter $\mathcal{S}$ stands for square and $\mathcal{C}$ stands for cube.) Then we construct $B=\bigoplus_{j=0}^{n-1} B_{j}$ with $B_{j}: \mathcal{C}[j] \rightarrow \mathcal{S}[j]$. Let us start the detailed construction. Define

$$
\begin{aligned}
\mathcal{S}[j]=\operatorname{span}\{v[3 j], v[3 j+1], v[3 j+2], \\
v[3 j+3 n], v[3 j+3 n+1], v[3 j+3 n+2]\}
\end{aligned}
$$

and

$$
\begin{aligned}
\mathcal{C}[j]=\operatorname{span}\{v[2 j], v[2 j+2 n], v[2 j+4 n], \\
v[2 j+1], v[2 j+2 n+1], v[2 j+4 n+1]\} .
\end{aligned}
$$

We will use the ordered base of $\mathcal{S}[j]$ (respectively $\mathcal{C}[j]$ ) as it appears in their definitions. Let $S_{j}$ (respectively $C_{j}$ ) be a restriction of $A$ to $\mathcal{S}[j]$ (respectively $\mathcal{C}[j])$. Observe that

$$
S_{j}=\omega^{3 j} \operatorname{diag}\left(1, \omega, \omega^{2},-1,-\omega,-\omega^{2}\right)
$$

and

$$
C_{j}=\omega^{2 j} \operatorname{diag}\left(1, \exp \left(\frac{2 \pi i}{3}\right), \exp \left(\frac{4 \pi i}{3}\right), \omega, \omega \exp \left(\frac{2 \pi i}{3}\right), \omega \exp \left(\frac{4 \pi i}{3}\right)\right) .
$$


Now, let $B \in \mathrm{U}(6 n)$ be any unitary of the form $B=\bigoplus_{j=0}^{n-1} B_{j}$ with unitary $B_{j}: \mathcal{C}[j] \rightarrow \mathcal{S}[j]$. We claim that

$$
\left\|B^{-1} A^{2} B-A^{3}\right\|_{\text {Frob }}=O_{\mathcal{U}}\left(\frac{1}{n}\right)
$$

Indeed,

$$
\left\|B^{-1} A^{2} B-A^{3}\right\|_{\mathrm{Frob}}^{2}=\sum_{j=0}^{n-1}\left\|B_{j}^{-1} S_{j}^{2} B_{j}-C_{j}^{3}\right\|_{\mathrm{Frob}}^{2},
$$

and we obtain

$$
\begin{aligned}
\left\|B_{j}^{-1} S_{j}^{2} B_{j}-C_{j}^{3}\right\|_{\text {Frob }}^{2} & =\left\|B_{j}^{-1}\left(S_{j}^{2}-\omega^{6 j}\right) B_{j}-\left(C_{j}^{3}-\omega^{6 j}\right)\right\|_{\text {Frob }}^{2} \\
& \leqslant\left\|S_{j}^{2}-\omega^{6 j}\right\|_{\text {Frob }}^{2}+\left\|C_{j}^{3}-\omega^{6 j}\right\|_{\text {Frob }}^{2} \\
& =2\left(\left|1-\omega^{2}\right|^{2}+\left|1-\omega^{4}\right|^{2}\right)+3\left|1-\omega^{3}\right|^{2}=O_{\mathcal{U}}\left(\frac{1}{n^{2}}\right),
\end{aligned}
$$

which entails the claim. Now, consider the unitary given by the matrix

$$
B_{j}=\frac{1}{\sqrt{2}}\left(\begin{array}{cccccc}
1 & 1 & 0 & 0 & 0 & 0 \\
1 & -1 & 0 & 0 & 0 & 0 \\
0 & 0 & 1 & 1 & 0 & 0 \\
0 & 0 & 1 & -1 & 0 & 0 \\
0 & 0 & 0 & 0 & 1 & 1 \\
0 & 0 & 0 & 0 & 1 & -1
\end{array}\right)
$$

and let

$$
\tilde{S}=\operatorname{diag}(1,1,1,-1,-1,-1)
$$

and

$$
\tilde{C}=\operatorname{diag}\left(1, \exp \left(\frac{2 \pi i}{3}\right), \exp \left(\frac{4 \pi i}{3}\right), 1, \exp \left(\frac{2 \pi i}{3}\right), \exp \left(\frac{4 \pi i}{3}\right)\right) .
$$

It is not hard to check that

$$
\left\|S_{j}-\omega^{3 j} \tilde{S}\right\|_{\text {Frob }}^{2}=O_{\mathcal{U}}\left(\frac{1}{n^{2}}\right)
$$

and

$$
\left\|C_{j}-\omega^{2 j} \tilde{C}\right\|_{\text {Frob }}^{2}=O_{\mathcal{U}}\left(\frac{1}{n^{2}}\right)
$$


Direct calculations show that $\left\|\tilde{C} B_{j}^{-1} \tilde{S} B_{j}-B_{j}^{-1} \tilde{S} B_{j} \tilde{C}\right\|_{\text {Frob }}^{2}=6$; so since

$$
\left\|A B^{-1} A B-B^{-1} A B A\right\|_{\mathrm{Frob}}^{2}=\sum_{j=0}^{n-1}\left\|C_{j} B_{j}^{-1} S_{j} B_{j}-B_{j}^{-1} S_{j} B_{j} C_{j}\right\|_{\mathrm{Frob}}^{2},
$$

the lemma follows.

2.3. An example of a finitely generated, nonresidually finite, Frobeniusapproximated group. Note that the example above provides a homomorphism into the ultraproduct $\varphi: \operatorname{BS}(2,3) \rightarrow \mathrm{U}_{\mathcal{U}}^{\|\cdot\| \|^{\text {rrob }}}$. The image $\Gamma=\varphi(\mathrm{BS}(2,3))$ is clearly Frobenius-approximated, but it is clearly not residually finite since, by construction, the elements $\varphi(a), \varphi(b) \in \Gamma$ satisfy (2.1) but not (2.2). In some sense, it is an artifact of the definitions that every non-Frobenius-stable group has a nontrivial Frobenius-approximated group quotient. It seems quite likely that the construction above is enough to show that $\mathrm{BS}(2,3)$ is itself Frobeniusapproximated. Indeed, even though the proof of this assertion is not spelled out in full detail in [42], it appears that Rădulescu's construction shows this. Note that it follows from the work of Kropholler [30] that $\operatorname{BS}(2,3)$ is residually solvable and hence MF; see [15].

\section{Diminishing the defect of asymptotic representations}

This section contains the key technical novelty of this article. We associate an element $[\alpha] \in H^{2}\left(\Gamma, \prod_{n \rightarrow \mathcal{U}}\left(\mathrm{M}_{k_{n}}(\mathbb{C}),\|\cdot\|\right)\right)$ to an asymptotic representation $\varphi_{n}: \Gamma \rightarrow \mathrm{U}\left(k_{n}\right)$. We prove that if $[\alpha]=0$, then the defect can be diminished in the sense that there is an equivalent asymptotic representation $\varphi_{n}^{\prime}$ with effectively better defect, more precisely $\operatorname{def}\left(\varphi_{n}^{\prime}\right)=o_{\mathcal{U}}\left(\operatorname{def}\left(\varphi_{n}\right)\right)$.

3.1. Assumptions for this section. For this section, we fix the following:

- A finitely presented group $\Gamma=\langle S \mid R\rangle$,

- a sequence of natural numbers $\left(k_{n}\right)_{n \in \mathbb{N}}$,

- a family of submultiplicative, unitarily invariant norms on $\mathrm{U}(k), k \in \mathbb{N}$, all denoted by $\|\cdot\|$, and

- an asymptotic representation $\varphi_{n}: S \rightarrow \mathrm{U}\left(k_{n}\right)$ with respect to the metrics associated with $\|\cdot\|$. 
Recall the ultraproduct notation introduced in Section 1.3, that is, $\mathrm{U}_{\mathcal{U}}^{\|\cdot\|}=$ $\prod_{n \rightarrow \mathcal{U}}\left(\mathrm{U}\left(k_{n}\right)\right.$, dist $\left._{\|\cdot\|}\right)$ and $\mathbf{M}_{\mathcal{U}}^{\|\cdot\|}=\prod_{n \rightarrow \mathcal{U}}\left(\mathbf{M}_{k_{n}}(\mathbb{C}),\|\cdot\|\right)$ and recall that since $\|\cdot\|$ is submultiplicative, $\mathrm{U}_{\mathcal{U}}^{\|\cdot\|}$ acts on $\mathrm{M}_{\mathcal{U}}^{\|\cdot\|}$ by multiplication. An asymptotic representation as above induces a homomorphism $\varphi_{\mathcal{U}}: \Gamma \rightarrow \mathrm{U}_{\mathcal{U}}^{\|\cdot\|}$ on the level of the group $\Gamma$. Thus, $\Gamma$ acts on $\mathrm{M}_{\mathcal{U}}^{\|\cdot\|}$ through $\varphi_{\mathcal{U}}$. With this in mind, we also want to fix the following:

- a section $\sigma: \Gamma \rightarrow \mathbb{F}_{S}$ of the natural surjection $\mathbb{F}_{S} \rightarrow \Gamma$, in particular, we have $\sigma(g) \sigma(h) \sigma(g h)^{-1} \in\langle R\rangle$ for all $g, h \in \Gamma$,

- a sequence $\tilde{\varphi}_{n}: \Gamma \rightarrow \mathrm{U}\left(k_{n}\right)$ such that $\tilde{\varphi}\left(1_{\Gamma}\right)=1_{k_{n}}, \tilde{\varphi}_{n}\left(g^{-1}\right)=\tilde{\varphi}_{n}(g)^{*}$, and for every $g \in \Gamma$,

$$
\left\|\varphi_{n}(\sigma(g))-\tilde{\varphi}_{n}(g)\right\|=O_{\mathcal{U}}\left(\operatorname{def}\left(\varphi_{n}\right)\right) .
$$

In particular, the sequence $\left(\tilde{\varphi}_{n}(g)\right)_{n \in \mathbb{N}}$ maps to the element $\varphi_{\mathcal{U}}(g)$ in the ultralimit, for all $g \in \Gamma$. (We say that $\tilde{\varphi}_{n}$ is a lift of $\varphi_{\mathcal{U}}$.)

For this, note that given any section $\sigma: \Gamma \rightarrow \mathbb{F}_{S}$, the sequence $\varphi_{n} \circ \sigma$ is a lift of $\varphi_{\mathcal{U}}$. There exists a section $\sigma$ with $\sigma\left(1_{\Gamma}\right)=1_{\mathbb{F}_{S}}$ and $\sigma\left(g^{-1}\right)=\sigma(g)^{-1}$ for all $g$ such that $g^{2} \neq 1_{\Gamma}$. We define $\tilde{\varphi}_{n}(g):=\varphi_{n}(\sigma(g))$ for all $g$ with $g^{2} \neq 1_{\Gamma}$. In the case $g^{2}=1_{\Gamma}$, by Lemma 1.13 , it holds that

$$
\left\|\varphi_{n}(\sigma(g))^{2}-1_{k_{n}}\right\|=O_{\mathcal{U}}\left(\operatorname{def}\left(\varphi_{n}\right)\right),
$$

so by Proposition 1.4, there are self-adjoint unitaries $B_{n} \in \mathrm{U}\left(k_{n}\right)$ such that

$$
\left\|B_{n}-\varphi_{n}(\sigma(g))\right\|=O_{\mathcal{U}}\left(\operatorname{def}\left(\varphi_{n}\right)\right) .
$$

By letting $\tilde{\varphi}_{n}(g):=B_{n}$, we get the desired map.

3.2. The cohomology class of an asymptotic representation. We want to define an element in $H^{2}\left(\Gamma, \mathrm{M}_{\mathcal{U}}^{\|\cdot\|}\right)$ associated with $\varphi_{n}$. To this end, we define $c_{n}:=$ $c_{n}\left(\varphi_{n}\right): \Gamma \times \Gamma \rightarrow \mathrm{M}_{k_{n}}(\mathbb{C})$ by

$$
c_{n}(g, h)=\frac{\tilde{\varphi}_{n}(g) \tilde{\varphi}_{n}(h)-\tilde{\varphi}_{n}(g h)}{\operatorname{def}\left(\varphi_{n}\right)},
$$

for all $n \in \mathbb{N}$ such that $\operatorname{def}\left(\varphi_{n}\right)>0$ and $c_{n}(g, h)=0$ otherwise, for all $g, h \in \Gamma$. The next proposition is a collection of basic properties of the maps $c_{n}$.

Proposition 3.1. Let $g, h, k \in \Gamma$. The maps $c_{n}$ satisfy the following equations:

$$
\tilde{\varphi}_{n}(g) c_{n}(h, k)-c_{n}(g h, k)+c_{n}(g, h k)-c_{n}(g, h) \tilde{\varphi}_{n}(k)=0,
$$




$$
c_{n}\left(g, g^{-1}\right)=c_{n}\left(1_{\Gamma}, g\right)=c_{n}\left(g, 1_{\Gamma}\right)=0 \quad \text { and } \quad c_{n}(g, h)^{*}=c_{n}\left(h^{-1}, g^{-1}\right) .
$$

Furthermore, we have for every $g, h \in \Gamma$,

$$
\left\|c_{n}(g, h)\right\|=O_{\mathcal{U}}(1)
$$

Proof. For all $g, h, k \in \Gamma$ and $n \in \mathbb{N}$, we have

$$
\begin{aligned}
\operatorname{def}\left(\varphi_{n}\right) \cdot\left(\tilde{\varphi}_{n}(g) c_{n}(h, k)-c_{n}(g h, k)+c_{n}(g, h k)-c_{n}(g, h) \tilde{\varphi}_{n}(k)\right) \\
=\tilde{\varphi}_{n}(g)\left(\tilde{\varphi}_{n}(h) \tilde{\varphi}_{n}(k)-\tilde{\varphi}_{n}(h k)\right)-\left(\tilde{\varphi}_{n}(g h) \tilde{\varphi}_{n}(k)-\tilde{\varphi}_{n}(g h k)\right) \\
\quad+\left(\tilde{\varphi}_{n}(g) \tilde{\varphi}_{n}(h k)-\tilde{\varphi}_{n}(g h k)\right)-\left(\tilde{\varphi}_{n}(g) \tilde{\varphi}_{n}(h)-\tilde{\varphi}_{n}(g h)\right) \tilde{\varphi}_{n}(k) \\
=\tilde{\varphi}_{n}(g) \tilde{\varphi}_{n}(h) \tilde{\varphi}_{n}(k)-\tilde{\varphi}_{n}(g) \tilde{\varphi}_{n}(h k)-\tilde{\varphi}_{n}(g h) \tilde{\varphi}_{n}(k)+\tilde{\varphi}_{n}(g h k) \\
\quad+\tilde{\varphi}_{n}(g) \tilde{\varphi}_{n}(h k)-\tilde{\varphi}_{n}(g h k)-\tilde{\varphi}_{n}(g) \tilde{\varphi}_{n}(h) \tilde{\varphi}_{n}(k)+\tilde{\varphi}_{n}(g h) \tilde{\varphi}_{n}(k) \\
=0,
\end{aligned}
$$

which proves the first equation. The second line of equations is immediate from the definition of $c_{n}$ and the fact that $\tilde{\varphi}_{n}\left(g^{-1}\right)=\tilde{\varphi}_{n}(g)^{*}$.

For the last assertion, note that since $\sigma(g) \sigma(h) \sigma(g h)^{-1} \in\langle R\rangle$, it follows from Lemma 1.13 that

$$
\left\|\varphi_{n}\left(\sigma(g) \sigma(h) \sigma(g h)^{-1}\right)-1_{k_{n}}\right\|=O_{\mathcal{U}}\left(\operatorname{def}\left(\varphi_{n}\right)\right) .
$$

Thus, it follows (by using Equation (3.1)) that

$$
\operatorname{def}\left(\varphi_{n}\right)\left\|c_{n}(g, h)\right\|=O_{\mathcal{U}}\left(\operatorname{def}\left(\varphi_{n}\right)\right) .
$$

Note for later use that for fixed $g, h \in \Gamma$, the bound in (3.2), which comes from Lemma 1.13, holds for all asymptotic representations $\varphi_{n}$ simultaneously (as long as we use the same presentation of $\Gamma$ and section $\sigma$ to construct $c_{n}$ ). In addition, Equation (3.2) entails that the sequence $c_{n}$ induces a map

$$
c=\left(c_{n}\right)_{n \in \mathbb{N}}: \Gamma \times \Gamma \rightarrow \mathrm{M}_{\mathcal{U}}^{\|\cdot\|} .
$$

This map is not a cocycle in the sense explained in Section 1.5, but, as the next corollary states, the map $\alpha(g, h):=c(g, h) \varphi_{\mathcal{U}}(g h)^{*}$ is. (The map $c$ is a cocycle in the equivalent picture of Hochschild cohomology and it turns out that some calculations are more natural with $c$, so we will also work with this map.) Even though we suppress it in the notation, keep in mind that $c$ and $\alpha$ depend on the lift $\tilde{\varphi}_{n}$ and on $\operatorname{def}\left(\varphi_{n}\right)$.

COROLlary 3.2. The map $\alpha: \Gamma \times \Gamma \rightarrow \mathrm{M}_{\mathcal{U}}^{\|\cdot\|}$ is a 2-cocycle with respect to the isometric action $\pi(g) T=\varphi_{\mathcal{U}}(g) T \varphi_{\mathcal{U}}(g)^{*}, g \in \Gamma, T \in \mathrm{M}_{\mathcal{U}}^{\|\cdot\|}$. 
Proof. Given $g, h, k \in \Gamma$, we have that

$$
\begin{aligned}
\varphi_{\mathcal{U}} & (g) \alpha(h, k) \varphi_{\mathcal{U}}(g)^{*}-\alpha(g h, k)+\alpha(g, h k)-\alpha(g, h) \\
= & \varphi_{\mathcal{U}}(g) c(h, k) \varphi_{\mathcal{U}}(h k)^{*} \varphi_{\mathcal{U}}(g)^{*}-c(g h, k) \varphi_{\mathcal{U}}(g h k)^{*} \\
& +c(g, h k) \varphi_{\mathcal{U}}(g h k)^{*}-c(g, h) \varphi_{\mathcal{U}}(g h)^{*} \\
= & \left(\varphi_{\mathcal{U}}(g) c(h, k)-c(g h, k)+c(g, h k)-c(g, h) \varphi_{\mathcal{U}}(k)\right) \varphi_{\mathcal{U}}(g h k)^{*} \\
= & 0,
\end{aligned}
$$

where we used that $\varphi_{\mathcal{U}}$ is a homomorphism and Proposition 3.1.

We call $\alpha$ the cocycle associated with the sequence $\left(\varphi_{n}\right)_{n \in \mathbb{N}}$.

Proposition 3.3. Assume that $\alpha$ represents the trivial cohomology class in $H^{2}\left(\Gamma, \mathbf{M}_{\mathcal{U}}^{\|\cdot\|}\right)$, that is, there exists a map $\beta: \Gamma \rightarrow \mathbf{M}_{\mathcal{U}}^{\|\cdot\|}$ satisfying

$$
\alpha(g, h)=\varphi_{\mathcal{U}}(g) \beta(h) \varphi_{\mathcal{U}}(g)^{*}-\beta(g h)+\beta(g), \quad g, h \in \Gamma .
$$

Then

$$
\begin{gathered}
\beta\left(1_{\Gamma}\right)=0, \\
\beta(g)=-\varphi_{\mathcal{U}}(g) \beta\left(g^{-1}\right) \varphi_{\mathcal{U}}(g)^{*} \\
c(g, h)=\varphi_{\mathcal{U}}(g) \beta(h) \varphi_{\mathcal{U}}(h)-\beta(g h) \varphi_{\mathcal{U}}(g h)+\beta(g) \varphi_{\mathcal{U}}(g h) .
\end{gathered}
$$

Furthermore, we can choose $\beta(g)$ to be skew-symmetric for all $g \in \Gamma$.

Proof. Equation (3.5) is equivalent to the equation from the assumption since $\alpha(g, h)=c(g, h) \varphi_{\mathcal{U}}(g h)^{*}$ for $g, h \in \Gamma$. Equation (3.3) follows from (3.5) and Proposition 3.1 with $g=h=1_{\Gamma}$ and (3.4) follows from (3.3), (3.5), and Proposition 3.1 with $h=g^{-1}$. For the last claim, we possibly need to alter $\beta$ a little. Note that $\beta^{\prime}(g):=-\beta(g)^{*}=\varphi_{\mathcal{U}}(g) \beta\left(g^{-1}\right)^{*} \varphi_{\mathcal{U}}(g)^{*}$ also satisfies (3.3)(3.5). Indeed,

$$
\begin{aligned}
c(g, h)= & c\left(h^{-1}, g^{-1}\right)^{*} \\
= & \left(\varphi_{\mathcal{U}}\left(h^{-1}\right) \beta\left(g^{-1}\right) \varphi_{\mathcal{U}}\left(g^{-1}\right)-\beta\left(h^{-1} g^{-1}\right) \varphi_{\mathcal{U}}\left(h^{-1} g^{-1}\right)\right. \\
& \left.+\beta\left(h^{-1}\right) \varphi_{\mathcal{U}}\left(h^{-1} g^{-1}\right)\right)^{*} \\
= & \varphi_{\mathcal{U}}(g) \beta\left(g^{-1}\right)^{*} \varphi_{\mathcal{U}}(h)-\varphi_{\mathcal{U}}(g h) \beta\left((g h)^{-1}\right)^{*}+\varphi_{\mathcal{U}}(g h) \beta\left(h^{-1}\right)^{*} \\
= & \beta^{\prime}(g) \varphi_{\mathcal{U}}(g h)-\beta^{\prime}(g h) \varphi_{\mathcal{U}}(g h)+\varphi_{\mathcal{U}}(g) \beta^{\prime}(h) \varphi_{\mathcal{U}}(h)
\end{aligned}
$$

for $g, h \in \Gamma$, which proves (3.5) whence the other two follow. Thus, replacing $\beta$ with

$$
\beta^{\sharp}(g):=\frac{\beta(g)-\beta(g)^{*}}{2}, \quad g \in \Gamma, n \in \mathbb{N},
$$


we see that $\beta^{\sharp}(g)$ is skew-symmetric and that Equations (3.3)-(3.5) are still satisfied.

3.3. Correction of the asymptotic representation. Now let $\beta$ be as above and let $\beta_{n}: \Gamma \rightarrow \mathrm{M}_{k_{n}}(\mathbb{C})$ be any skew-symmetric lift of $\beta$. Then $\exp \left(-\operatorname{def}\left(\varphi_{n}\right) \beta_{n}(g)\right)$ is a unitary for every $g \in \Gamma$, so we can define a sequence of maps $\psi_{n}: \Gamma \rightarrow \mathrm{U}\left(k_{n}\right)$ by

$$
\psi_{n}(g)=\exp \left(-\operatorname{def}\left(\varphi_{n}\right) \beta_{n}(g)\right) \tilde{\varphi}_{n}(g) .
$$

Note that since $\tilde{\varphi}_{n}\left(1_{\Gamma}\right)=1_{k_{n}}$ and $\beta_{n}\left(1_{\Gamma}\right)=0$, we have $\psi_{n}\left(1_{\Gamma}\right)=1_{k_{n}}$.

In the proofs of Proposition 3.4 and Lemma 3.5, we will make use of two basic inequalities that hold for any $k \in \mathbb{N}$ and $A \in \mathrm{M}_{k}(\mathbb{C})$ :

$$
\begin{gathered}
\left\|1_{k}-\exp (A)\right\| \leqslant\|A\| \exp (\|A\|) \\
\left\|1_{k}+A-\exp (A)\right\| \leqslant\|A\|^{2} \exp (\|A\|) .
\end{gathered}
$$

They are simple consequences of the definition $\exp (A)=\sum_{k=0}^{\infty} \frac{A^{k}}{k !}$ and the triangle inequality and submultiplicativity of the norm.

Proposition 3.4. With the notation from above, for every $g \in \Gamma$, we have

$$
\left\|\tilde{\varphi}_{n}(g)-\psi_{n}(g)\right\|=O_{\mathcal{U}}\left(\operatorname{def}\left(\varphi_{n}\right)\right) .
$$

More precisely,

$$
\left\|\tilde{\varphi}_{n}(g)-\psi_{n}(g)\right\| \leqslant 2\left\|\beta_{n}(g)\right\| \operatorname{def}\left(\varphi_{n}\right)
$$

for most $n \in \mathbb{N}$.

Proof. Let $g \in \Gamma$. By unitary invariance and submultiplicativity, we get that

$$
\begin{aligned}
\left\|\tilde{\varphi}_{n}(g)-\psi_{n}(g)\right\| & =\left\|1_{k_{n}}-\exp \left(-\operatorname{def}\left(\varphi_{n}\right) \beta_{n}(g)\right)\right\| \\
& \stackrel{(3.6)}{\leqslant} \operatorname{def}\left(\varphi_{n}\right)\left\|\beta_{n}(g)\right\| \exp \left(\operatorname{def}\left(\varphi_{n}\right)\left\|\beta_{n}(g)\right\|\right)
\end{aligned}
$$

and since $\left\|\beta_{n}(g)\right\|$ is a bounded sequence and $\lim _{n \rightarrow \mathcal{U}} \operatorname{def}\left(\varphi_{n}\right)=0$, we have $\exp \left(\operatorname{def}\left(\varphi_{n}\right)\left\|\beta_{n}(g)\right\|\right) \leqslant 2$ for most $n$ and the result follows.

It follows that $\left.\psi_{n}\right|_{S}$ is an asymptotic representation satisfying $\operatorname{def}\left(\left.\psi_{n}\right|_{S}\right)=$ $O_{\mathcal{U}}\left(\operatorname{def}\left(\varphi_{n}\right)\right)$, but we prove that the defect is actually $o_{\mathcal{U}}\left(\operatorname{def}\left(\varphi_{n}\right)\right)$.

LEMMA 3.5. For any $g, h \in \Gamma$, we have that

$$
\left\|\psi_{n}(g h)-\psi_{n}(g) \psi_{n}(h)\right\|=o_{\mathcal{U}}\left(\operatorname{def}\left(\varphi_{n}\right)\right)
$$


Proof. Let $\xi_{n}(x):=\left(1_{k_{n}}-\operatorname{def}\left(\varphi_{n}\right) \beta_{n}(x)\right) \tilde{\varphi}_{n}(x)$, for $x \in \Gamma$, and let $g, h \in \Gamma$ be fixed. Let $C=2 \max _{x \in\{g, h, g h\}}\|\beta(x)\|$. Whence it follows that for most $n \in \mathbb{N}$,

$$
\left\|\psi_{n}(x)-\xi_{n}(x)\right\| \stackrel{(3.7)}{\leqslant} C \cdot \operatorname{def}\left(\varphi_{n}\right)^{2},
$$

for $x \in\{g, h, g h\}$. By the above (and by submultiplicativity), it follows that

$$
\left\|\psi_{n}(g h)-\psi_{n}(g) \psi_{n}(h)\right\|=\left\|\xi_{n}(g h)-\xi_{n}(g) \xi_{n}(h)\right\|+o_{\mathcal{U}}\left(\operatorname{def}\left(\varphi_{n}\right)\right),
$$

so it suffices to show that

$$
\left\|\xi_{n}(g h)-\xi_{n}(g) \xi_{n}(h)\right\|=o_{\mathcal{U}}\left(\operatorname{def}\left(\varphi_{n}\right)\right)
$$

which amounts to the following calculations:

$$
\begin{aligned}
\xi_{n}(g h) & -\xi_{n}(g) \xi_{n}(h) \\
= & \tilde{\varphi}_{n}(g h)-\tilde{\varphi}_{n}(g) \tilde{\varphi}_{n}(h) \\
& +\operatorname{def}\left(\varphi_{n}\right)\left(-\beta_{n}(g h) \tilde{\varphi}_{n}(g h)+\tilde{\varphi}_{n}(g) \beta_{n}(h) \tilde{\varphi}_{n}(h)+\beta_{n}(g) \tilde{\varphi}_{n}(g) \tilde{\varphi}_{n}(h)\right) \\
& -\operatorname{def}\left(\varphi_{n}\right)^{2} \beta_{n}(g) \tilde{\varphi}_{n}(g) \beta_{n}(h) \tilde{\varphi}(h) \\
= & \operatorname{def}\left(\varphi_{n}\right)\left(-c_{n}(g, h)\right. \\
& \left.+\tilde{\varphi}_{n}(g) \beta_{n}(h) \tilde{\varphi}_{n}(h)-\beta_{n}(g h) \tilde{\varphi}_{n}(g h)+\beta_{n}(g) \tilde{\varphi}_{n}(g) \tilde{\varphi}_{n}(h)\right) \\
& -\operatorname{def}\left(\varphi_{n}\right)^{2} \beta_{n}(g) \tilde{\varphi}_{n}(g) \beta_{n}(h) \tilde{\varphi}_{n}(h) .
\end{aligned}
$$

By Equation (3.5), the expression

$$
-c_{n}(g, h)+\tilde{\varphi}_{n}(g) \beta_{n}(h) \tilde{\varphi}_{n}(h)-\beta_{n}(g h) \tilde{\varphi}_{n}(g h)+\beta_{n}(g) \tilde{\varphi}_{n}(g) \tilde{\varphi}_{n}(h)
$$

tends to zero. Furthermore, using submultiplicativity of the norm, we conclude $\left\|\beta_{n}(g) \tilde{\varphi}_{n}(g) \beta_{n}(h) \tilde{\varphi}_{n}(h)\right\|$ is bounded, and we reach the desired conclusion.

At last, we define the asymptotic representation $\varphi_{n}^{\prime}: S \rightarrow \mathrm{U}\left(k_{n}\right)$ by $\varphi_{n}^{\prime}=\left.\psi_{n}\right|_{S}$ and reach the desired conclusion $\operatorname{def}\left(\varphi_{n}^{\prime}\right)=o_{\mathcal{U}}\left(\operatorname{def}\left(\varphi_{n}\right)\right)$. Let us, for reference's sake, formulate the result properly.

THEOREM 3.6. Let $\Gamma=\langle S \mid R\rangle$ be a finitely presented group and let $\varphi_{n}: S \rightarrow \mathrm{U}\left(k_{n}\right)$ be an asymptotic representation with respect to a family of submultiplicative, unitarily invariant norms. Assume that the associated 2cocycle $\alpha=\alpha\left(\varphi_{n}\right)$ is trivial in $H^{2}\left(\Gamma, \mathrm{M}_{\mathcal{U}}^{\|\cdot\|}\right)$. Then there exists an asymptotic representation $\varphi_{n}^{\prime}: S \rightarrow \mathrm{U}\left(k_{n}\right)$ such that

(i) $\operatorname{dist}\left(\varphi_{n}, \varphi_{n}^{\prime}\right)=O_{\mathcal{U}}\left(\operatorname{def}\left(\varphi_{n}\right)\right)$ and 
(ii) $\operatorname{def}\left(\varphi_{n}^{\prime}\right)=o_{\mathcal{U}}\left(\operatorname{def}\left(\varphi_{n}\right)\right)$.

Proof. We adopt the above notation. Assertion (i) follows from Proposition 3.4; let $r=x_{1} x_{2} \cdots x_{m} \in R$ be written as a reduced word, where $x_{j} \in S \cup S^{-1}, j=1$, $\ldots, m$. By iteration of Lemma 3.5 (using that $\psi_{n}$ takes unitary values and that $\|\cdot\|$ is unitarily invariant), we see that

$$
\begin{aligned}
\left\|\varphi_{n}^{\prime}(r)-1_{k_{n}}\right\| & =\left\|\psi_{n}\left(x_{1}\right) \psi_{n}\left(x_{2}\right) \cdots \psi_{n}\left(x_{m}\right)-1_{k_{n}}\right\| \\
& =\left\|\psi_{n}\left(x_{1} x_{2}\right) \psi_{n}\left(x_{3}\right) \cdots \psi_{n}\left(x_{m}\right)-1_{k_{n}}\right\|+o_{\mathcal{U}}\left(\operatorname{def}\left(\varphi_{n}\right)\right) \\
& \vdots \\
& =\left\|\psi\left(1_{\Gamma}\right)-1_{k_{n}}\right\|+o_{\mathcal{U}}\left(\operatorname{def}\left(\varphi_{n}\right)\right) .
\end{aligned}
$$

Since $\psi\left(1_{\Gamma}\right)=1_{k_{n}}$, we are done.

The converse of Theorem 3.6 is also valid in the following sense.

Proposition 3.7. Let $\Gamma=\langle S \mid R\rangle$ be a finitely presented group and let $\varphi_{n}$, $\psi_{n}: S \rightarrow \mathrm{U}\left(k_{n}\right)$ be asymptotic representations with respect to some family of submultiplicative, unitarily invariant norms and suppose

- $\operatorname{dist}\left(\varphi_{n}, \psi_{n}\right)=O_{\mathcal{U}}\left(\operatorname{def}\left(\varphi_{n}\right)\right)$ and

- $\operatorname{def}\left(\psi_{n}\right)=o_{\mathcal{U}}\left(\operatorname{def}\left(\varphi_{n}\right)\right)$.

Then, the 2-cocycle $\alpha$ associated with $\left(\varphi_{n}\right)_{n \in \mathbb{N}}$ is trivial in $H^{2}\left(\Gamma, \mathbf{M}_{\mathcal{U}}^{\|\cdot \cdot\|}\right)$. In particular, if $\varphi_{n}$ is sufficiently close to a homomorphism, $\alpha$ is trivial.

Proof. If $\operatorname{def}\left(\varphi_{n}\right)=0$ for most $n \in \mathbb{N}$, there is nothing to prove, so let us assume this is not the case. Let $\tilde{\varphi}_{n}, \tilde{\psi}_{n}: \Gamma \rightarrow \mathrm{U}\left(k_{n}\right)$ be the induced maps we get by fixing a section $\Gamma \rightarrow \mathbb{F}_{S}$ as explained in the beginning of this section. We note that the sequences $\tilde{\varphi}_{n}$ and $\tilde{\psi}_{n}$ induce the same map $\varphi_{\mathcal{U}}$ in the limit. Define

$$
\gamma_{n}(g)=\frac{\tilde{\varphi}_{n}(g)-\tilde{\psi}_{n}(g)}{\operatorname{def}\left(\varphi_{n}\right)}
$$

for $n$ with $\operatorname{def}\left(\varphi_{n}\right)>0$ and $\gamma_{n}(g)=0$ otherwise. By the first bullet in our assumptions, $\gamma_{n}$ is essentially bounded, so it defines an element $\gamma(g) \in \mathbf{M}_{\mathcal{U}}^{\|\cdot\|}$. If we prove that

$$
c(g, h)=\varphi_{\mathcal{U}}(g) \gamma(h)-\gamma(g h)+\gamma(g) \varphi_{\mathcal{U}}(h),
$$


it will follow easily that $\beta(g):=\gamma(g) \varphi_{\mathcal{U}}(g)^{*}$ will satisfy $d \beta=\alpha$. First note that it follows from the second bullet in our assumptions that for every $g, h \in \Gamma$

$$
\left\|\tilde{\psi}_{n}(g h)-\tilde{\psi}_{n}(g) \tilde{\psi}_{n}(h)\right\|=o_{\mathcal{U}}\left(\operatorname{def}\left(\varphi_{n}\right)\right)
$$

thus,

$$
\begin{aligned}
\operatorname{def}\left(\varphi_{n}\right) \cdot\left(\tilde{\varphi}_{n}(g) \gamma_{n}(h)\right. & \left.-\gamma_{n}(g h)+\gamma_{n}(g) \tilde{\psi}_{n}(h)\right) \\
= & \tilde{\varphi}_{n}(g) \tilde{\varphi}_{n}(h)-\tilde{\varphi}_{n}(g) \tilde{\psi}_{n}(h)-\tilde{\varphi}_{n}(g h)+\tilde{\psi}_{n}(g h) \\
& +\tilde{\varphi}_{n}(g) \tilde{\psi}_{n}(h)-\tilde{\psi}_{n}(g) \tilde{\psi}_{n}(h) \\
= & \tilde{\varphi}_{n}(g) \tilde{\varphi}_{n}(h)-\tilde{\varphi}_{n}(g h)+\tilde{\psi}_{n}(g h)-\tilde{\psi}_{n}(g) \tilde{\psi}_{n}(h) \\
= & \operatorname{def}\left(\varphi_{n}\right) \cdot c_{n}(g, h)+o_{\mathcal{U}}\left(\operatorname{def}\left(\varphi_{n}\right)\right) .
\end{aligned}
$$

Now the result follows by dividing by $\operatorname{def}\left(\varphi_{n}\right)$ (which is possible for most $n$ ) and taking the limit.

It is now clear that we are in need of large classes of groups for which general vanishing results for the second cohomology with Banach or Hilbert space coefficients can be proven. This will be the subject of the next section. But first let us mention an alternative approach that can be used to prove Theorem 3.6.

3.4. Asymptotic representations and extensions. As mentioned in Section 1.5, the second cohomology characterizes extensions of $\Gamma$ with abelian kernel and that in this picture, coboundaries correspond to splitting extensions. Thus, Theorem 3.6 and Corollary 3.7 show that finding the improved $\varphi_{n}^{\prime}$ is equivalent to finding a splitting for a certain extension. The connection between asymptotic representations and extensions can be seen directly without going through the above computations, and this idea can actually be used to prove Theorem 3.6. Since this approach is very illustrative (it shows, for instance, very clearly what rôle submultiplicativity plays), we sketch the proof.

We retain the assumptions from Section 3.1 and introduce some more notation. Letting $\varepsilon_{n}:=\operatorname{def}\left(\varphi_{n}\right)$, for $n \in \mathbb{N}$, we define

$$
\mathrm{N}\left(O_{\mathcal{U}}\left(\varepsilon_{n}\right)\right)=\left\{\left(u_{n}\right)_{n \in \mathbb{N}} \in \prod_{n \in \mathbb{N}} \mathrm{U}\left(k_{n}\right) \mid\left\|u_{n}-1_{k_{n}}\right\|=O_{\mathcal{U}}\left(\varepsilon_{n}\right)\right\}
$$

and

$$
\mathrm{U}\left(O_{\mathcal{U}}\left(\varepsilon_{n}\right)\right)=\prod_{n \in \mathbb{N}} \mathrm{U}\left(k_{n}\right) / N\left(O_{\mathcal{U}}\left(\varepsilon_{n}\right)\right)
$$


Similarly, we define $\mathrm{N}\left(o_{\mathcal{U}}\left(\varepsilon_{n}\right)\right)$ and $\mathrm{U}\left(o_{\mathcal{U}}\left(\varepsilon_{n}\right)\right)$. We saw that the asymptotic representation $\left(\varphi_{n}\right)_{n \in \mathbb{N}}$ induces a homomorphism $\varphi_{\mathcal{U}}: \Gamma \rightarrow \mathrm{U}_{\mathcal{U}}^{\|\cdot\|}$, but Lemma 1.13 actually implies the existence of an induced homomorphism

$$
\hat{\varphi}_{\mathcal{U}}: \Gamma \rightarrow \mathrm{U}\left(O_{\mathcal{U}}\left(\varepsilon_{n}\right)\right) .
$$

Now we observe that the existence of $\varphi_{n}^{\prime}: S \rightarrow \mathrm{U}\left(k_{n}\right)$ with $\operatorname{dist}\left(\varphi_{n}, \varphi_{n}^{\prime}\right)=$ $O_{\mathcal{U}}\left(\operatorname{def}\left(\varphi_{n}\right)\right)$ and $\operatorname{def}\left(\varphi_{n}^{\prime}\right)=o_{\mathcal{U}}\left(\operatorname{def}\left(\varphi_{n}\right)\right)$ as in Theorem 3.6 is equivalent to the existence of a lift $\varphi_{\mathcal{U}}^{\prime}$ of $\hat{\varphi}_{\mathcal{U}}$ :

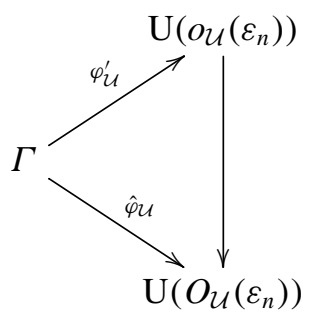

We also see that the map $\hat{\varphi}_{\mathcal{U}}$ fits into the following commutative diagram:

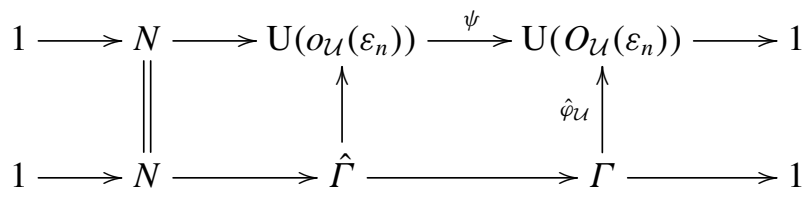

where $\hat{\Gamma}$ is the pullback through $\hat{\varphi}$ and $\psi$ and

$$
N:=\mathrm{N}\left(O_{\mathcal{U}}\left(\varepsilon_{n}\right)\right) / \mathrm{N}\left(o_{\mathcal{U}}\left(\varepsilon_{n}\right)\right)
$$

Combining these two observations, it easily follows that $\varphi_{n}$ can be improved to $\varphi_{n}^{\prime}$ if and only if the bottom row in the latter diagram splits. Now, since $\|\cdot\|$ is submultiplicative, the group $N$ is actually abelian. Indeed, for all $T, S \in \mathrm{U}(k)$, we have that

$$
\begin{aligned}
\left\|T S T^{*} S^{*}-1_{k}\right\| & =\|T S-S T\| \\
& =\left\|\left(T-1_{k}\right)\left(S-1_{k}\right)-\left(S-1_{k}\right)\left(T-1_{k}\right)\right\| \\
& \leqslant 2\left\|T-1_{k}\right\|\left\|S-1_{k}\right\|,
\end{aligned}
$$

so if $\left(T_{n}\right)_{n \in \mathbb{N}},\left(S_{n}\right)_{n \in \mathbb{N}} \in N\left(O\left(\varepsilon_{n}\right)\right)$, then

$$
\left(T_{n} S_{n} T_{n}^{*} S_{n}^{*}\right)_{n \in \mathbb{N}} \in N\left(O\left(\varepsilon_{n}^{2}\right)\right) \subseteq N\left(o\left(\varepsilon_{n}\right)\right),
$$


whence the claim follows. Hence, as explained in Section 1.5, the extension $1 \rightarrow$ $N \rightarrow \hat{\Gamma} \rightarrow \Gamma \rightarrow 1$ corresponds to an element $[\hat{\alpha}] \in H^{2}(\Gamma, N)$, and we conclude that $\varphi_{n}$ can be improved if and only if $[\hat{\alpha}]=[0]$. Now, the coefficients $N$ are not exactly the same as $\mathbf{M}_{\mathcal{U}}^{\|\cdot\|}$ in Theorem 3.6, but with a little effort, one can prove that $N$ is a real Banach space (or a real Hilbert space in the case $\|\cdot\|=\|\cdot\|_{\text {Frob }}$ ) with an isometric $\Gamma$-action and the existence of an equivariant homomorphism $\theta: N \rightarrow \mathrm{M}_{\mathcal{U}}^{\|\cdot\|}$ such that $[\theta \circ \hat{\alpha}]=[\alpha]$.

REMARK 3.8. We note that this approach also works for the most part if $\|\cdot\|$ is not submultiplicative. In this case, however, the group $N$ is not abelian and the second cohomology with non-abelian coefficients is much less tractable in general.

This alternative approach to the problem at hand is rather conceptual and elegant, and the proof that we chose to present in detail also has its merits. The cocycle $\alpha$ can be computed directly from $\left(\varphi_{n}\right)_{n \in \mathbb{N}}$, and in cases where the associated 1-cochain $\beta$ can be computed explicitly from $\alpha$, this gives us an explicit expression for $\varphi_{n}^{\prime}$.

\section{Cohomology vanishing and examples of $\boldsymbol{n}$-Kazhdan groups}

Recall that if $\Gamma$ is a finitely (or, more generally, compactly) generated group, then $\Gamma$ has Kazhdan's property (T) if and only if the first cohomology $H^{1}\left(\Gamma, \mathcal{H}_{\pi}\right)=0$ for every unitary representation $\pi: \Gamma \rightarrow \mathrm{U}\left(\mathcal{H}_{\pi}\right)$ on a Hilbert space $\mathcal{H}_{\pi}$; see [9] for a proof and more background information. We will consider groups for which the higher cohomology groups vanish. Higher dimensional vanishing phenomena have been studied in various articles; see, for example, $[5,6,12,20,21,35,37]$.

We propose the following terminology.

Definition 4.1. Let $n \in \mathbb{N}$. A group $\Gamma$ is called $n$-Kazhdan if $H^{n}\left(\Gamma, \mathcal{H}_{\pi}\right)$ vanishes for all unitary representations $\left(\pi, \mathcal{H}_{\pi}\right)$ of $\Gamma$. We call $\Gamma$ strongly $n$ Kazhdan, if $\Gamma$ is $k$-Kazhdan for $k=1, \ldots, n$.

So 1-Kazhdan is the Kazhdan's classical property (T). See [5, 35] for discussions of other related higher dimensional analogues of property (T). It will be central in our proof that by an application of the open mapping theorem, vanishing of cohomology with Hilbert space coefficients implies that cocycles are coboundaries with control on the norms. This is explained in the following proposition and its corollary, where we use the terminology introduced in Equation (1.1). 
Proposition 4.2. Let $n \in \mathbb{N}$, let $\Gamma$ be a countable group, let $\pi: \Gamma \rightarrow \mathrm{U}\left(\mathcal{H}_{\pi}\right)$ be a unitary representation, and assume that $H^{n}\left(\Gamma, \mathcal{H}_{\pi}\right)=\{0\}$. Then for every finite set $F \subseteq \Gamma^{n-1}$, there exist a finite set $F_{\pi} \subseteq \Gamma^{n}$ and a constant $C_{\pi, F} \geqslant 0$ such that for every cocycle $z \in Z^{n}\left(\Gamma, \mathcal{H}_{\pi}\right)$, there is an element $b \in C^{n-1}\left(\Gamma, \mathcal{H}_{\pi}\right)$ such that $z=d^{n-1} b$ and $\|b\|_{F}<C_{\pi, F}\|z\|_{F_{\pi}}$.

Proof. By definition of the topology on $C^{n}\left(\Gamma, \mathcal{H}_{\pi}\right)$, the basic open sets are given by

$$
U_{\delta, F^{\prime}}=\left\{f \in C^{n}\left(\Gamma, \mathcal{H}_{\pi}\right) \mid\|f\|_{F^{\prime}}<\delta\right\},
$$

for a finite $F^{\prime} \subseteq \Gamma^{n}$ and $\delta>0$. Since the map $d^{n-1}: C^{n-1}\left(\Gamma, \mathcal{H}_{\pi}\right) \rightarrow Z^{n}\left(\Gamma, \mathcal{H}_{\pi}\right)$ is linear, bounded, and surjective, the open mapping theorem applies (see [46]); so there are $C_{\pi, F}>0$ and $F_{\pi} \subseteq \Gamma^{n}$ such that

$$
U_{C_{\pi, F}^{-1}, F_{\pi}} \cap Z^{n}\left(\Gamma, \mathcal{H}_{\pi}\right) \subseteq d^{n-1}\left(U_{1, F}\right) .
$$

In other words, if $z \in Z^{n}\left(\Gamma, \mathcal{H}_{\pi}\right),\|z\|_{F_{\pi}}=1$, then $C_{\pi, F}^{-1} z \in U_{C_{\pi, F}^{-1}, F_{\pi}}$; so there is $b \in C^{n-1}\left(\Gamma, \mathcal{H}_{\pi}\right)$ such that $d^{n-1} b=z$ and $\|b\|_{F}<C_{\pi, F}=C_{\pi, F}\|z\|$. This proves the claim.

We need the fact that if $H^{2}\left(\Gamma, \mathcal{H}_{\pi}\right)$ vanishes universally, the set $F_{\pi}$ and the bound $C_{\pi, F}$ can be chosen universally for all unitary representations $\pi$. This is the consequence of an easy diagonalization argument.

COROLlaRY 4.3. Let $n \in \mathbb{N}$ and $\Gamma$ be a countable $n$-Kazhdan group. Then for every finite set $F \subseteq \Gamma^{n}$, there are a finite set $F_{0} \subseteq \Gamma^{n-1}$ and a constant $C_{F} \geqslant 0$ such that for all unitary representations $\pi$ of $\Gamma$ and all cocycles $z \in Z^{n}\left(\Gamma, \mathcal{H}_{\pi}\right)$, there is an element $b \in C^{n-1}\left(\Gamma, \mathcal{H}_{\pi}\right)$ such that $z=d^{n-1} b$ and $\|b\|_{F}<C_{F}\|z\|_{F_{0}}$.

We also observe the following extension proposition.

Proposition 4.4. Consider a short exact sequence of groups

$$
1 \rightarrow \Lambda \rightarrow \tilde{\Gamma} \rightarrow \Gamma \rightarrow 1
$$

If $\Lambda$ is strongly $n$-Kazhdan and $\Gamma$ is $n$-Kazhdan, then $\tilde{\Gamma}$ is also $n$-Kazhdan. In particular, this applies if $\Lambda$ or $\Gamma$ is finite.

Proof. By the Hochschild-Serre spectral sequence [13], it is enough to show that $H^{k}\left(\Gamma, H^{l}\left(\Lambda, \mathcal{H}_{\left.\pi\right|_{\Lambda}}\right)\right)$ vanishes for all $k, l \in \mathbb{N}$ with $k+l=n$. If $l>0$, then $H^{l}(\Lambda$, $\left.\mathcal{H}_{\left.\pi\right|_{\Lambda}}\right)$ vanishes. For $l=0$ and $k=n$, we have $H^{0}\left(\Lambda, \mathcal{H}_{\left.\pi\right|_{\Lambda}}\right)=\operatorname{Fix}\left(\left.\pi\right|_{\Lambda}\right)$ (the set of fixed vectors in $\mathcal{H}_{\pi \mid \Lambda}$ ), which is a Hilbert space, and the induced action of $\Gamma$ is a unitary representation; so we conclude that $H^{n}\left(\Gamma, H^{0}\left(\Lambda, \mathcal{H}_{\left.\pi\right|_{\Lambda}}\right)\right)$ vanishes. 
In view of the previous section, it is natural to ask if there exists a nonresidually finite group such that $H^{2}(\Gamma, A)$ vanishes for all $C^{*}$-algebras $A$ whenever $\Gamma$ acts on $A$ by automorphisms. We are not able to answer this question; however, one can show that $H^{1}\left(\Gamma, \ell^{\infty}(\Gamma)\right)$ does not vanish for any infinite group, which makes a positive answer somewhat unlikely. Here, we view $\ell^{\infty}(G)$ as a $G$-module with respect to the right translation action. Indeed, let $d: \Gamma \times \Gamma \rightarrow \mathbb{N}$ be a proper leftinvariant metric. Then, $c(g):=\left(h \mapsto d\left(1_{\Gamma}, h\right)-d\left(1_{\Gamma}, h g\right)\right)_{h \in \Gamma}$ defines a cocycle $c: \Gamma \rightarrow \ell^{\infty}(\Gamma)$ which cannot be the boundary of an element in $\ell^{\infty}(\Gamma)$ if $\Gamma$ is infinite.

4.1. Higher rank $p$-adic lattices are 2-Kazhdan. Finally, this section provides examples, or every $n \geqslant 2$, of groups which are $n$-Kazhdan. The results are essentially known and we recall them in detail for convenience.

Let $K$ be a non-archimedean local field of residue class $q$, that is, if $\mathcal{O} \subset K$ is the ring of integers and $\mathfrak{m} \subset \mathcal{O}$ is its unique maximal ideal, then $q=|\mathcal{O} / \mathfrak{m}|$. Let $\mathbf{G}$ be a simple $K$-algebraic group of $K$-rank $r$ and assume that $r \geqslant 1$. The group $G:=\mathbf{G}(K)$ acts on the associated Bruhat-Tits building $\mathcal{B}$. For more information on the theory of buildings, see [1]. The latter is an infinite, contractible, pure simplicial complex of dimension $r$, on which $G$ acts transitively on the chambers, that is, the top-dimensional simplices. Let $\Gamma$ be a uniform lattice in $G$, that is, a discrete cocompact subgroup of $G$. When $\Gamma$ is also torsion-free (which can always be achieved by replacing $\Gamma$ by a finite-index subgroup), the quotient $X:=\Gamma \backslash \mathcal{B}$ is a finite $r$-dimensional simplicial complex and $\Gamma=\pi_{1}(X)$. In particular, the group $\Gamma$ is finitely presented. We will use the following theorem which essentially appears in the work of Ballmann and Światkowski [6] building on previous work of Garland [24].

THEOREM 4.5. For every natural number $r \geqslant 2$, there exists $q_{0}(r) \in \mathbb{N}$ such that the following holds. If $q \geqslant q_{0}(r)$ and $G$ and $\Gamma$ are as above, then $\Gamma$ is strongly $(r-1)-K a z h d a n$. In particular, if $r \geqslant 3$, then $\Gamma$ is 2-Kazhdan.

Recall that being 1-Kazhdan is equivalent to Kazhdan's property (T). As it is well known, $G$ and $\Gamma$ as above have property (T) for every $r>2$ and for all $q$. With this in mind, we find it plausible that one can take $q_{0}(r)=2$ in the above theorem.

Note that such $\Gamma$ contains a finite-index torsion-free group $\Lambda$. Proposition 4.4 implies that it suffices to prove that $\Lambda$ is $(r-1)$-Kazhdan. So one can assume that $\Gamma$ is torsion-free.

Theorem 4.5 for finite-dimensional Hilbert spaces is Theorem 8.3 in the seminal paper of Garland [24]. The general case is stated in the last paragraph of 
Section 3.1 on page 631 in the work of Ballmann-Światkowski [6]. It is deduced from Theorem 2.5 there that theorem asserts á la Garland [24] that the desired cohomology vanishing follows from sharp estimates of the spectral gap of the local Laplacians, that is, the Laplacians of the proper links of the complex. These estimates (called also p-adic curvature) are given in [24, Lemmas 6.3 and 8.2]. So altogether Theorem 4.5 is proven. The method and estimates of Garland are used also in $[37,55]$ and more recently in $[28,35]$.

Let us give the reader just a notational warning: when we say rank (following the common practice nowadays), we mean the $K$-rank of $G$ as a $p$-adic group (and we denoted it by $r$ ) and then it follows that the dimension of the associated Bruhat-Tits building is equal to $r$. Garland refers to the rank of the Tits system which, in his notation, he denotes $l+1$. Hence, our $r$ is equal to his $l$.

It is very natural to wonder what happens in the analogous real case. It is worth noting that already $H^{5}\left(\mathrm{SL}_{n}(\mathbb{Z}), \mathbb{R}\right)$ is nontrivial for $n$ large enough [11]; thus $\mathrm{SL}_{n}(\mathbb{Z})$ fails to be 5-Kazhdan for $n$ large enough. Similarly, note that $H^{2}(\operatorname{Sp}(2 n$, $\mathbb{Z}), \mathbb{R})=\mathbb{R}$ for all $n \geqslant 2$ [11] so that the natural generalization to higher rank lattices in real Lie groups has to be formulated carefully, maybe just by excluding an explicit list of finite-dimensional unitary representations.

QUESTION 4.6. Is $\mathrm{SL}_{n}(\mathbb{Z})$ 2-Kazhdan (at least for large $\left.n\right)$ ?

\section{Proofs of the main results}

In order to finish the proofs of Theorem 1.1 and Theorem 1.2, we need to show that finitely presented 2-Kazhdan groups are Frobenius-stable and that some of them are not residually finite. The main result follows then from Corollary 5.4 and the constructions in Section 5.2.

5.1. The Frobenius stability of 2-Kazhdan groups. We now consider 2Kazhdan groups and asymptotic representations with respect to the Frobenius norm. As $\prod_{n \rightarrow \mathcal{U}}\left(\mathrm{M}_{k_{n}}(\mathbb{C}),\|\cdot\|_{\text {Frob }}\right)$ is a Hilbert space, the techniques of Section 3 can be applied and the defect of every asymptotic representation can be diminished. We start by completing the proof of Theorem 1.2.

THEOREM 5.1. Let $\Gamma$ be a finitely presented group. If $\Gamma$ is 2-Kazhdan, then it is Frobenius-stable.

Proof. Let $\Gamma=\langle S \mid R\rangle$. As mentioned, the ultraproduct

$$
\mathrm{M}_{\mathcal{U}}^{\|\cdot\|_{\text {Frob }}}:=\prod_{n \rightarrow \mathcal{U}}\left(\mathrm{M}_{k_{n}}(\mathbb{C}),\|\cdot\|_{\text {Frob }}\right)
$$


is a Hilbert space and $\Gamma$ acts on this space by invertible isometries, that is, by unitaries; so $H^{2}\left(\Gamma, \mathbf{M}_{\mathcal{U}}^{\|\cdot\|_{\text {Frob }}}\right)$ vanishes. In particular, the 2-cocycles defined in Section 3 are always trivial. As noted, the bounds from Equation (3.2) hold simultaneously for all asymptotic representations. This, combined with Corollary 4.3 , shows that there is a constant $C$ such that for all asymptotic representations $\varphi_{n}: \Gamma \rightarrow \mathrm{U}\left(k_{n}\right)$ with respect to $\|\cdot\|_{\text {Frob }}$, we can choose the 1-cochain $\beta$ associated with $\alpha$ so that it satisfies

$$
2 \max _{s \in S}\|\beta(s)\|_{\text {Frob }} \leqslant C .
$$

Define the quantity

$$
\theta(\varphi):=\operatorname{HomDist}(\varphi)-2 C \operatorname{def}(\varphi)
$$

for any map $\varphi: S \rightarrow \mathrm{U}(k)$ (for any $k \in \mathbb{N}$ ). We note that if $\varphi_{n}: S \rightarrow \mathrm{U}\left(k_{n}\right)$ is any asymptotic representation, then $\lim _{n \rightarrow \mathcal{U}} \theta\left(\varphi_{n}\right) \geqslant 0$ and equality holds if and only if $\varphi_{n}$ is equivalent to a sequence of homomorphisms.

Now we fix a sequence $\left(\varepsilon_{n}\right)_{n \in \mathbb{N}}$ of strictly positive real numbers such that $\lim _{n \rightarrow \mathcal{U}} \varepsilon_{n}=0$ and let $\left(k_{n}\right)_{n \in \mathbb{N}}$ be a sequence of natural numbers. By the above, we need to prove that for all sequences of $\varepsilon_{n}$-almost representations $\psi_{n}: S \rightarrow$ $\mathrm{U}\left(k_{n}\right)$, the quantity $\theta\left(\psi_{n}\right)$ tends to 0 . For each $n \in \mathbb{N}$, the set of $\varepsilon_{n}$-almost homomorphisms $\varphi: S \rightarrow \mathrm{U}\left(k_{n}\right)$ is compact and since $\theta$ is continuous, there is $\varphi_{n}: S \rightarrow \mathrm{U}\left(k_{n}\right)$ such that $\operatorname{def}\left(\varphi_{n}\right) \leqslant \varepsilon_{n}$ and $\varphi_{n}$ maximizes $\theta$ for all $n \in \mathbb{N}$. Evidently, $\varphi_{n}$ is an asymptotic representation. Thus, by Proposition 3.4 and Theorem 3.6, there is an asymptotic representation $\varphi_{n}^{\prime}: S \rightarrow \mathrm{U}\left(k_{n}\right)$ such that $\operatorname{dist}\left(\varphi_{n}, \varphi_{n}^{\prime}\right) \leqslant C \operatorname{def}\left(\varphi_{n}\right)$ and

$$
\operatorname{def}\left(\varphi_{n}^{\prime}\right) \leqslant \frac{1}{4} \operatorname{def}\left(\varphi_{n}\right)
$$

for most $n \in \mathbb{N}$. In particular, $\varphi_{n}^{\prime}$ is also an $\varepsilon_{n}$-almost representation, and it follows that for most $n$, we have

$$
\operatorname{HomDist}\left(\varphi_{n}\right) \leqslant \operatorname{HomDist}\left(\varphi_{n}^{\prime}\right)+C \operatorname{def}\left(\varphi_{n}\right) .
$$

Furthermore, by maximality, we have that

$$
\operatorname{HomDist}\left(\varphi_{n}^{\prime}\right)-2 C \operatorname{def}\left(\varphi_{n}^{\prime}\right)=\theta\left(\varphi_{n}^{\prime}\right) \leqslant \theta\left(\varphi_{n}\right)=\operatorname{HomDist}\left(\varphi_{n}\right)-2 C \operatorname{def}\left(\varphi_{n}\right),
$$

and putting these estimates together, we get

$$
\operatorname{HomDist}\left(\varphi_{n}^{\prime}\right)-2 C \operatorname{def}\left(\varphi_{n}^{\prime}\right) \leqslant \operatorname{HomDist}\left(\varphi_{n}^{\prime}\right)-C \operatorname{def}\left(\varphi_{n}\right)
$$

or

$$
\operatorname{def}\left(\varphi_{n}\right) \stackrel{(5.2)}{\leqslant} 2 \operatorname{def}\left(\varphi_{n}^{\prime}\right) \stackrel{(5.1)}{\leqslant} \frac{1}{2} \operatorname{def}\left(\varphi_{n}\right)
$$


which can only be the case if $\operatorname{def}\left(\varphi_{n}\right)=0$ for most $n$. But then, $\varphi_{n}$ is really a representation for most $n \in \mathbb{N}$; so $\operatorname{HomDist}\left(\varphi_{n}\right)=0$ and we conclude $\lim _{n \rightarrow \mathcal{U}} \theta\left(\varphi_{n}\right)=0$. Since $\theta\left(\varphi_{n}\right)$ was chosen maximal, we conclude that $\lim _{n \rightarrow \mathcal{U}} \theta\left(\psi_{n}\right)=0$ for all $\varepsilon_{n}$-almost representations $\psi_{n}$.

REMARK 5.2. Small modifications of the proof show that the result is still valid if one replaces $\|\cdot\|_{\text {Frob }}$ with any submultiplicative norm $\|\cdot\|$ and the 2Kazhdan assumption with a suitable cohomology vanishing assumption. To prove Corollary 4.3 for other classes of Banach space coefficients, we need that the class in question is stable under the ultraproduct Banach space construction from Section 1.3. However, this extra condition is always satisfied, as it can be proved that ultraproducts of the spaces $M_{\mathcal{U}}^{\|\cdot\|}$ are isometrically isomorphic to $M_{\mathcal{V}}^{\|\cdot\|}$ for a (possibly) different ultrafilter $\mathcal{V}$.

This, for instance, gives a sufficient condition for stability with respect to the operator norm, where one could assume vanishing of second cohomology with coefficients in a $C^{*}$-algebra, but it seems difficult to prove the existence of a group $\Gamma$ with such properties - a task that will already occupy the remaining sections in the Hilbert space case.

REMARK 5.3. Note that Theorem 5.1 together with Proposition 4.4 imply that virtually free groups are Frobenius-stable-a fact that seems cumbersome to establish directly.

For the sake of reference, we formulate the following dichotomy, which is an immediate corollary to Theorem 5.1, explicitly.

COROllary 5.4. Let $\Gamma$ be a finitely presented 2-Kazhdan group. Then either

- $\Gamma$ is residually finite or

- $\Gamma$ is not Frobenius-approximated.

The techniques in Section 3 rely on submultiplicativity of the norm and thus cannot be directly applied to the normalized Hilbert-Schmidt norm $\|\cdot\|_{\mathrm{HS}}$. It is worth noting, though, that since $\frac{1}{\sqrt{k}}\|A\|_{\text {Frob }}=\|A\|_{\mathrm{HS}} \leqslant\|A\|_{\mathrm{op}} \leqslant\|A\|_{\text {Frob }}$ for $A \in$ $\mathrm{M}_{k}(\mathbb{C})$, we get the following immediate corollary to Theorem 5.1.

COROLlaRY 5.5. Let $\Gamma=\langle S \mid R\rangle$ be a finitely presented 2-Kazhdan group and let $\varphi_{n}: S \rightarrow \mathrm{U}\left(k_{n}\right)$ be a sequence of maps such that

$$
\operatorname{def}\left(\varphi_{n}\right)=o_{\mathcal{U}}\left(k_{n}^{-1 / 2}\right)
$$


where the defect is measured with respect to either $\|\cdot\|_{\mathrm{HS}}$ or $\|\cdot\|_{\mathrm{op}}$. Then $\varphi_{n}$ is equivalent to a sequence of homomorphisms.

Proof. Let $\|\cdot\|$ be the norm in question. Then

$$
\operatorname{def}_{\|\cdot\|_{\text {Frob }}}\left(\varphi_{n}\right) \leqslant \sqrt{k_{n}} \operatorname{def}_{\|\cdot\|}\left(\varphi_{n}\right)=o_{\mathcal{U}}(1) .
$$

In other words, $\varphi_{n}$ is an asymptotic representation with respect to $\|\cdot\|_{\text {Frob }}$; so by Theorem 5.1, there are representations $\pi_{n}: \Gamma \rightarrow \mathrm{U}\left(k_{n}\right)$ with

$$
\left\|\varphi_{n}(s)-\pi_{n}(s)\right\| \leqslant\left\|\varphi_{n}(s)-\pi_{n}(s)\right\|_{\text {Frob }}=o_{\mathcal{U}}(1)
$$

for $s \in S$.

The preceding corollary provides some quantitative information about the Connes' Embedding Problem. Indeed, if a finitely presented, nonresidually finite, 2-Kazhdan group is $\mathrm{U}_{\mathrm{HS}}$-approximated, then there is some upper bound on the quality of the approximation in terms of the dimension of the unitary group. Needless to say, it would be very interesting to decide if groups as above are $\mathrm{U}_{\mathrm{HS}}$-approximated.

5.2. Nonresidually finite $\mathbf{2}$-Kazhdan groups. In this section, we present examples of finitely presented, nonresidually finite groups which are 2-Kazhdan and hence finish the proof of Theorem 1.1. Note first that all the examples $\Gamma$ presented in Section 4.1 are residually finite. In this section, we will show that some of these $\Gamma$ 's have finite central extensions

$$
1 \rightarrow C \rightarrow \tilde{\Gamma} \rightarrow \Gamma \rightarrow 1
$$

where $C$ is a finite cyclic group and $\tilde{\Gamma}$ is not residually finite. Now, $C$ being finite is strongly $n$-Kazhdan for every $n$, and so, if $\Gamma$ is 2-Kazhdan, then the same holds for $\tilde{\Gamma}$ by Proposition 4.4. Hence, we may combine our results of this section with the results from the previous section to obtain examples of 2-Kazhdan groups which are not residually finite.

Our construction will imitate the construction of Deligne [18] of nonresidually finite central extensions of some nonuniform arithmetic lattices in real Lie groups. See also the work of Raghunathan $[43,44]$, where such central extensions were constructed for some uniform lattices in $\operatorname{Spin}(2, n)$. These examples were later used by Toledo [50] in his famous work showing the existence of algebraic varieties with nonresidually finite fundamental groups. A short and very readable exposition of Deligne's argument was given by Witte Morris (see http://people. uleth.ca/ dave.morris/talks/deligne-torsion.pdf). 
Our examples are $p$-adic analogues of Deligne's examples and his original proof actually works for them. He assumed the algebraic group $\mathbf{G}$ to be isotropic and hence got only nonuniform lattices as, at the time, the congruence subgroup property was known only in such cases. Nowadays, we can argue for more general lattices along the same lines.

Let $D$ be the standard quaternion algebra over $\mathbb{Z}$, defined as

$$
D=\mathbb{Z}\langle i, j, k\rangle /\left(i^{2}=j^{2}=k^{2}=-1, i j=k\right)
$$

and set $D_{R}:=R \otimes_{\mathbb{Z}} D$ for an arbitrary unital commutative ring $R$. It is well known that $D_{\mathbb{R}}$ is the Hamiltonian division algebra $\mathbb{H}$, whereas $D_{\mathbb{Q}_{p}} \cong \mathrm{M}_{2}\left(\mathbb{Q}_{p}\right)$ for $p \geqslant 3$, where the second isomorphism is basically a consequence of the fact that the congruence $x^{2}+y^{2}=-1$ can be solved modulo $p$. Consider also the standard involution $\tau: D_{R} \rightarrow D_{R}$ and let $h: D_{R}^{n} \times D_{R}^{n} \rightarrow D_{R}$ be the canonical sesquilinear hermitian form on $D_{R}^{n}$, that is,

$$
h\left(\left(x_{1}, \ldots, x_{n}\right),\left(y_{1}, \ldots, y_{n}\right)\right)=x_{1} \tau\left(y_{1}\right)+\cdots+x_{n} \tau\left(y_{n}\right) .
$$

Consider now $\mathbf{G}(R):=\mathrm{SU}\left(n, D_{R}, h\right)$. Note that $\mathbf{G}(R)$ is simply the group formed by those $n \times n$-matrices with entries in $D_{R}$ such that the associated $D_{R}$-linear map preserves the form $h$. The functor $\mathbf{G}$ is an absolutely almost simple, simply connected $\mathbb{Q}$-algebraic group which is $\overline{\mathbb{Q}}$-isomorphic to $\mathbf{S p}(2 n)$ and hence of type $C_{n}$; see [39, Section 2.3]. Embedding $D_{\mathbb{R}} \subset M_{2}(\mathbb{C})$, one can show that $\mathbf{G}(\mathbb{R})$ is isomorphic to a simply connected compact Lie group of type $C_{n}$, namely the quaternionic unitary group $\operatorname{Sp}(n)=\mathrm{U}(2 n) \cap \operatorname{Sp}(2 n, \mathbb{C})$.

Let now $p \geqslant 3$ be a rational prime. Since $D\left(\mathbb{Q}_{p}\right) \cong \mathrm{M}_{2}\left(\mathbb{Q}_{p}\right)$, the group $\mathbf{G}$ becomes split over $\mathbb{Q}_{p}$ and $\mathbf{G}\left(\mathbb{Q}_{p}\right)$ is a noncompact group isomorphic to $\operatorname{Sp}(2 n$, $\left.\mathbb{Q}_{p}\right)$. The group $\Gamma:=\mathbf{G}(\mathbb{Z}[1 / p])$ sits diagonally as a lattice in $\mathbf{G}(\mathbb{R}) \times \mathbf{G}\left(\mathbb{Q}_{p}\right)$. However, since $\mathbf{G}(\mathbb{R})$ is compact, this yields that

$$
\Gamma=\mathbf{G}(\mathbb{Z}[1 / p]) \subset \mathbf{G}\left(\mathbb{Q}_{p}\right)
$$

is also a lattice. It is a standard fact that lattices in $\operatorname{Sp}\left(2 n, \mathbb{Q}_{p}\right)$ are cocompact, basically since $\operatorname{Sp}\left(2 n, \mathbb{Q}_{p}\right)$ admits a basis of neighborhoods of the identity that consists of torsion-free subgroups. In this concrete case, we can identify $\Gamma$ with the group

$$
\mathrm{U}(2 n) \cap \operatorname{Sp}(2 n, \mathbb{Z}[i, 1 / p]) .
$$

It was proved by Rapinchuk [45] and Tomanov [51] that the group $\Gamma=$ $\mathbf{G}(\mathbb{Z}[1 / p])$ has the congruence subgroup property. Let us explain what this means in the adelic language: The group $\Gamma$ is a subgroup of $\mathbf{G}(\mathbb{Q})$ and we can define two topologies on $\mathbf{G}(\mathbb{Q})$ as follows. The first is the arithmetic topology, 
for which the arithmetic subgroups, that is, the subgroups commensurable to $\Gamma$ serve as a fundamental system of neighborhoods of the identity. The second is the congruence topology for which we take as a basis of neighborhoods of the identity only those arithmetic groups which contain, for some natural number $m$ with $(m, p)=1$, one of the principal congruence subgroups

$$
\Gamma(m):=\operatorname{ker}(\mathbf{G}(\mathbb{Z}[1 / p]) \rightarrow \mathbf{G}(\mathbb{Z} / m \mathbb{Z})) .
$$

We denote by $\widehat{\mathbf{G}(\mathbb{Q})}$ the completion with respect to the arithmetic topology and by $\overline{\mathbf{G}(\mathbb{Q})}$ the completion with respect to the congruence topology. There is a canonical surjective homomorphism

$$
\pi: \widehat{\mathbf{G}(\mathbb{Q})} \rightarrow \overline{\mathbf{G}(\mathbb{Q})} .
$$

The result of Rapinchuk and Tomanov $[45,51]$ combined with the work of PrasadRapinchuk [41] says that in our case, $\pi$ is an isomorphism of topological groups.

Now, by the strong approximation theorem, $\overline{\mathbf{G}(\mathbb{Q})}$ is isomorphic to

$$
\mathbf{G}\left(\mathbb{A}_{f \backslash\{p\}}\right)=\prod_{l \neq p}^{*} \mathbf{G}\left(\mathbb{Q}_{l}\right),
$$

where $\prod^{*}$ denotes the restricted product as usual and $\mathbb{A}_{f \backslash\{p\}}$ is a subring of the $\mathbb{Q}$-adeles $\mathbb{A}$, the restricted product of $\mathbb{Q}_{l}$ for all primes $l \neq p$. In particular, we get

$$
\mathbf{G}(\mathbb{A})=\mathbf{G}(\mathbb{R}) \times \mathbf{G}\left(\mathbb{Q}_{p}\right) \times \overline{\mathbf{G}(\mathbb{Q})} .
$$

Now a result of Prasad [40] (see also Deodhar [19] and Deligne [18]) says that for every $p, \mathbf{G}\left(\mathbb{Q}_{p}\right)$ has a universal central extension

$$
1 \rightarrow C(p) \rightarrow \widetilde{\mathbf{G}\left(\mathbb{Q}_{p}\right)} \rightarrow \mathbf{G}\left(\mathbb{Q}_{p}\right) \rightarrow 1,
$$

where $C(p)$ denotes the group of roots of unity in $\mathbb{Q}_{p}$, that is, a cyclic group of order $p-1$. We denote by $\widetilde{\Gamma}$ and by $\widetilde{\mathbf{G}(\mathbb{Q})}$ the inverse images of $\Gamma$ and $\mathbf{G}(\mathbb{Q})$ under the quotient map in the above extension.

We claim that if $p \geqslant 5$, then the group $\widetilde{\Gamma}$ is not residually finite.

Proposition 5.6. Every finite-index subgroup of $\widetilde{\Gamma}$ contains the unique subgroup of index 2 in $C(p)$. In particular, if $p \geqslant 5, \widetilde{\Gamma}$ is not residually finite.

Proof. To prove this, we will lift the arithmetic topology from $\mathbf{G}(\mathbb{Q})$ to its central extension $\widetilde{\mathbf{G}(\mathbb{Q})}$ as follows. We define the arithmetic topology on $\widetilde{\mathbf{G}(\mathbb{Q})}$ as the topology for which all subgroups commensurable to $\widetilde{\Gamma}$ serve as a fundamental 
system of neighborhoods of the identity. We denote by $\widehat{\widehat{\mathbf{G}(\mathbb{Q})}}$ its Hausdorff completion. It is clear from the definition that there exists a central extension of topological groups

$$
1 \rightarrow Z \rightarrow \widehat{\widehat{\mathbf{G}(\mathbb{Q})}} \rightarrow \widehat{\mathbf{G}(\mathbb{Q})} \rightarrow 1,
$$

where $Z$ is a quotient of $C(p)$, say by the quotient homomorphism $\mu: C(p) \rightarrow Z$, where $\operatorname{ker}(\mu)$ is exactly the intersection of all the finite-index subgroups of $\widetilde{\Gamma}$. The ultimate goal is to show that if $p \geqslant 5$, then $\operatorname{ker}(\mu)$ is nontrivial which would show that $\widetilde{\Gamma}$ is not residually finite. Define now

$$
\widetilde{E}:=\mathbf{G}(\mathbb{R}) \times \widetilde{\mathbf{G}\left(\mathbb{Q}_{p}\right)} \times \widehat{\widehat{\mathbf{G}(\mathbb{Q})}}
$$

and observe that it maps onto $\mathbf{G}(\mathbb{R}) \times \mathbf{G}\left(\mathbb{Q}_{p}\right) \times \widehat{\mathbf{G}(\mathbb{Q})}=\mathbf{G}(\mathbb{A})$ with kernel $1 \times$ $C(p) \times Z$. Finally, we set

$$
E=\frac{\widetilde{E}}{\{(1, a, b) \in 1 \times C(p) \times Z \mid b=\mu(a)\}} .
$$

Now, the group $E$ is a central extension of $\mathbf{G}(\mathbb{A})$ with kernel isomorphic to $Z$. Moreover, we also see from the definitions that the natural diagonal map $\widetilde{\mathbf{G}(\mathbb{Q})} \rightarrow$ $\widetilde{E} \rightarrow E$ sends $a \in C(p) \subset \widetilde{\mathbf{G}(\mathbb{Q})}$ to $(1, a, \mu(a))$ and hence factors through a homomorphism $\mathbf{G}(\mathbb{Q}) \rightarrow E$. This shows that the central extension

$$
1 \rightarrow Z \rightarrow E \rightarrow \mathbf{G}(\mathbb{A}) \rightarrow 1
$$

splits over the subgroup $\mathbf{G}(\mathbb{Q})$ of $\mathbf{G}(\mathbb{A})$. Note that since $\mathbf{G}(\mathbb{Q})$ is perfect, the same applies to $\mathbf{G}(\mathbb{A})$. Then a result on $\mathbf{G}(\mathbb{A})$ going back to Moore [33] for split groups and Prasad-Rapinchuk [41] for the general case asserts that the universal central extension of $\mathbf{G}(\mathbb{A})$ that splits over $\mathbf{G}(\mathbb{Q})$ has, in the case of our $\mathbf{G}$, a kernel of order 2-basically since the groups of roots of unity in $\mathbb{Q}$ is $\{ \pm 1\}$. Hence, we can conclude that $|Z| \leqslant 2$. This proves the first part. More specifically, this shows that the kernel of the map from the profinite completion $\widehat{\widetilde{\Gamma}}$ of $\widetilde{\Gamma}$, which is realized as a compact-open subgroup of $\widehat{\widehat{\mathbf{G}(\mathbb{Q})}}$, to the profinite completion $\widehat{\Gamma}$ of $\Gamma$, which is realized as a compact-open subgroup of $\widehat{\mathbf{G}(\mathbb{Q})}=\overline{\mathbf{G}(\mathbb{Q})}$, is of order at most 2 . Hence, every finite-index subgroup of $\widetilde{\Gamma}$ contains the index 2 subgroup of $C(p)$ in the center of $\widetilde{\Gamma}$.

Now, if $p \geqslant 5$, then $2<p-1$, and this proves that $\widetilde{\Gamma}$ is not residually finite.

In conclusion, since $\widetilde{\Gamma}$ is 2 -Kazhdan, by Theorem 4.5 and Proposition 4.4 , it cannot be Frobenius-approximated by Corollary 5.4. This finishes the proof of Theorem 1.1. 


\section{Acknowledgements}

The first and the last authors were supported by ERC Consolidator Grant No. 681207. The second author was supported by the ERC when visiting the Hebrew University at Jerusalem. The third author was supported by the ERC, NSF, and BSF. The results of this article are part of the PhD project of the first author.

The last author wants to thank David Fisher for fruitful discussions back in March 2011 - the idea of a cohomological obstruction to stability of asymptotic representations was first found there and later independently by the second and third author. We are grateful to Pierre Pansu and especially to Andrei Rapinchuk for useful remarks and references. We thank the Isaac Newton Institute in Cambridge for its hospitality during the workshop Approximation, deformation, and quasification (supported by EPSRC Grant No. EP/K032208/1) as part of the program on Non-positive curvature: group actions and cohomology.

Sincere thanks to the unnamed referee for many useful remarks and comments.

\section{Conflict of Interest: None.}

\section{References}

[1] P. Abramenko and K. S. Brown, 'Theory and applications', in Buildings, Graduate Texts in Mathematics, 248 (Springer, New York, 2008).

[2] R. Bhatia, Matrix Analysis, Graduate Texts in Mathematics, 169 (Springer, New York, 1997).

[3] G. Arzhantseva, 'Asymptotic approximations of finitely generated groups', in Extended Abstracts Fall 2012-Automorphisms of Free Groups, Trends Math. Res. Perspect. CRM Barc., 1 (Springer, Cham, 2014), 7-15.

[4] G. Arzhantseva and L. Păunescu, 'Almost commuting permutations are near commuting permutations', J. Funct. Anal. 269(3) (2015), 745-757.

[5] U. Bader and P. W. Nowak, 'Cohomology of deformations', J. Topol. Anal. 7(1) (2015), 81-104.

[6] W. Ballmann and J. Światkowski, 'On $L^{2}$-cohomology and property (T) for automorphism groups of polyhedral cell complexes', Geom. Funct. Anal. 7(4) (1997), 615-645.

[7] G. Baumslag and D. Solitar, 'Some two-generator one-relator non-Hopfian groups', Bull. Amer. Math. Soc. 68 (1962), 199-201.

[8] O. Becker, A. Lubotzky and A. Thom, 'Stability and invariant random subgroups', Duke Math. J. 168(12) (2019), 2207-2234.

[9] B. Bekka, P. de la Harpe and A. Valette, Kazhdan's Property (T), New Mathematical Monographs, 11 (Cambridge University Press, Cambridge, 2008).

[10] B. Blackadar and E. Kirchberg, 'Generalized inductive limits of finite-dimensional $C^{*}$ algebras', Math. Ann. 307(3) (1997), 343-380.

[11] A. Borel, 'Stable real cohomology of arithmetic groups', Ann. Sci. Éc. Norm. Supér. (4) 7(1974) (1975), 235-272. 
[12] A. Borel and N. Wallach, Continuous Cohomology, Discrete Subgroups, and Representations of Reductive Groups, 2nd edn, Mathematical Surveys and Monographs, 67 (American Mathematical Society, Providence, RI, 2000).

[13] K. S. Brown, Cohomology of Groups, Graduate Texts in Mathematics, 87 (Springer, New York, 1994), Corrected reprint of the 1982 original.

[14] M. Burger, N. Ozawa and A. Thom, 'On Ulam stability', Israel J. Math. 193(1) (2013), 109-129.

[15] J. Carrion, M. Dadarlat and C. Eckhardt, 'On groups with quasidiagonal $C^{*}$-algebras', J. Funct. Anal. 265(1) (2013), 135-152.

[16] A. Connes, 'Classification of injective factors. Cases $I I_{1} I I_{\infty} I I I_{\lambda} \lambda \neq 1$ ', Ann. of Math. (2) 104(1) (1976), 73-115.

[17] M. De Chiffre, N. Ozawa and A. Thom, 'Operator algebraic approach to inverse and stability theorems for amenable groups’, Mathematika 65(1) (2019), 98-118.

[18] P. Deligne, 'Extensions centrales non résiduellement finies de groupes arithmétiques', $C$. $R$. Acad. Sci. Paris Sér. A-B 287(4) (1978), A203-A208. (French, with English summary).

[19] V. V. Deodhar, 'On central extensions of rational points of algebraic groups', Bull. Amer. Math. Soc. 81 (1975), 573-575.

[20] J. Dymara and T. Januszkiewicz, 'New Kazhdan groups', Geom. Dedicata 80(1-3) (2000), 311-317.

[21] J. Dymara and T. Januszkiewicz, 'Cohomology of buildings and their automorphism groups', Invent. Math. 150(3) (2002), 579-627.

[22] R. Exel and T. Loring, 'Almost commuting unitary matrices', Proc. Amer. Math. Soc. 106(4) (1989), 913-915.

[23] T. Fritz, 'On infinite-dimensional state spaces', J. Math. Phys. 54(5)052107 (2013).

[24] H. Garland, ' $p$-adic curvature and the cohomology of discrete subgroups of $p$-adic groups', Ann. of Math. (2) 97 (1973), 375-423.

[25] L. Glebsky and L. M. Rivera, 'Sofic groups and profinite topology on free groups', J. Algebra 320(9) (2008), 3512-3518.

[26] M. Gromov, 'Endomorphisms of symbolic algebraic varieties', J. Eur. Math. Soc. 1(2) (1999), 109-197.

[27] K. Grove, H. Karcher and E. A. Ruh, 'Jacobi fields and Finsler metrics on compact Lie groups with an application to differentiable pinching problems', Math. Ann. 211 (1974), 7-21.

[28] A. Gundert and U. Wagner, 'On eigenvalues of random complexes', Israel J. Math. 216(2) (2016), 545-582.

[29] D. Kazhdan, 'On E-representations', Israel J. Math. 43(4) (1982), 315-323.

[30] P. H. Kropholler, 'Baumslag-Solitar groups and some other groups of cohomological dimension two', Comment. Math. Helv. 65(4) (1990), 547-558.

[31] A. Lubotzky, 'High dimensional expanders', Proc. Int. Cong. Math. 1 (2018), 705-730.

[32] A. Lubotzky and I. Oppenheim, 'Non p-norm approximated groups', J. d'Anal. Math., to appear.

[33] C. C. Moore, 'Group extensions of $p$-adic and adelic linear groups', Publ. Math. Inst. Hautes Etudes Sci. 35 (1968), 157-222.

[34] N. Nikolov, J. Schneider and A. Thom, 'Some remarks on finitarily approximated groups', J. Éc. Polytech. Math. 5 (2018), 239-258.

[35] I. Oppenheim, 'Vanishing of cohomology and property (T) for groups acting on weighted simplicial complexes', Groups Geom. Dyn. 9(1) (2015), 67-101. 
[36] N. Ozawa, M. Rørdam and Y. Sato, 'Elementary amenable groups are quasidiagonal', Geom. Funct. Anal. 25(1) (2015), 307-316.

[37] P. Pansu, 'Formules de Matsushima, de Garland et propriété (T) pour des groupes agissant sur des espaces symétriques ou des immeubles', Bull. Soc. Math. France 126(1) (1998), 107-139. (French, with English and French summaries).

[38] V. G. Pestov, 'Hyperlinear and sofic groups: a brief guide', Bull. Symb. Logic 14(4) (2008), 449-480.

[39] V. Platonov and A. S. Rapinchuk, Algebraic Groups and Number Theory, Pure and Applied Mathematics 139 (Academic Press, Inc., Boston, MA, 1994).

[40] G. Prasad, 'Deligne's topological central extension is universal', Adv. Math. 181(1) (2004), 160-164.

[41] G. Prasad and A. S. Rapinchuk, 'Computation of the metaplectic kernel', Publ. Math. Inst. Hautes Etudes Sci. 84 (1997), 91-187.

[42] F. Rădulescu, 'The von Neumann algebra of the non-residually finite Baumslag group $\langle a$, $b\left|a b^{3} a^{-1}=b^{2}\right\rangle$ embeds into $R^{\omega}$, in Hot Topics in Operator Theory, Theta Ser. Adv. Math., 9 (Theta, Bucharest, 2008), 173-185.

[43] M. S. Raghunathan, 'Torsion in cocompact lattices in coverings of Spin(2, n)', Math. Ann. 266(4) (1984), 403-419.

[44] M. S. Raghunathan, 'Corrigendum: "Torsion in cocompact lattices in coverings of Spin(2, n)"', Math. Ann. 303(3) (1995), 575-578.

[45] A. S. Rapinchuk, 'On the congruence subgroup problem for algebraic groups', Dokl. Akad. Nauk SSSR 306(6) (1989), 1304-1307 (Russian); English transl., Soviet Math. Dokl. 39 (1989), no. 3, 618-621.

[46] W. Rudin, Functional Analysis, 2nd edn, International Series in Pure and Applied Mathematics (McGraw-Hill, Inc., New York, 1991).

[47] A. Tikuisis, S. White and W. Winter, 'Quasidiagonality of nuclear $C^{*}$-algebras', Ann. of Math. (2) 185(1) (2017), 229-284.

[48] A. Thom, 'Finitary approximations of groups and their applications', Proc. Int. Cong. Math. 2 (2018), 1775-1796.

[49] A. Thom, 'Examples of hyperlinear groups without factorization property', Groups Geom. Dyn. 4(1) (2010), 195-208.

[50] D. Toledo, 'Projective varieties with non-residually finite fundamental group', Publ. Math. Inst. Hautes Etudes Sci. 77 (1993), 103-119.

[51] G. Tomanov, 'On the congruence-subgroup problem for some anisotropic algebraic groups over number fields', J. Reine Angew. Math. 402 (1989), 138-152.

[52] A. M. Turing, 'Finite approximations to Lie groups', Ann. of Math . (2) 39(1) (1938), 105-111.

[53] S. Ulam, A Collection of Mathematical Problems, Interscience Tracts in Pure and Applied Mathematics, 8 (Interscience Publishers, New York-London, 1960).

[54] D. Voiculescu, 'Asymptotically commuting finite rank unitary operators without commuting approximants', Acta Sci. Math. (Szeged) 45(1-4) (1983), 429-431.

[55] A. Żuk, 'Property (T) and Kazhdan constants for discrete groups', Geom. Funct. Anal. 13(3) (2003), 643-670. 